\title{
Magnetization steps in a diluted Heisenberg antiferromagnetic chain: Theory and experiments on TMMC:Cd
}

\author{
A. Paduan-Filho* N. F. Oliveira, Jr., and V. Bindilatt \\ Instituto de Física, Universidade de São Paulo, \\ C.P. 66.318, 05315-970 São Paulo-SP, Brazil
}

\author{
S. Foner \\ Francis Bitter Magnet Laboratory, Massachusetts Institute of Technology, Cambridge, MA 02139 \\ Y. Shapira \\ Department of Physics and Astronomy, Tufts University, Medford, MA 02155
}

(Dated: November 6, 2018)

\begin{abstract}
A theory for the equilibrium low-temperature magnetization $M$ of a diluted Heisenberg antiferromagnetic chain is presented. Only the nearest-neighbor exchange interaction is included, and the distribution of the magnetic ions is assumed to be random. Values of the magnetic fields $B_{i}$ at the magnetization steps (MST's) from finite chains with 2 to 5 spins (pairs, triplets, quartets, and quintets) are given for chains composed of spins $S=5 / 2$. The magnitudes of these MST's as a function of the fraction, $\mathrm{x}$, of cations that are magnetic are given for any $S$. An expression for the apparent saturation value of $M$ is derived. The magnetization curve, $M$ versus $B$, is calculated using the exact contributions of finite chains with 1 to 5 spins, and the "rise and ramp approximation" for longer chains. An expression for the low-temperature saturation magnetic field $B_{s}(n)$ of a finite chain with $n$ spins is given. Some non-equilibrium effects that occur in a rapidly changing $B$, are also considered. Some of these result from the absence of thermal equilibrium within the sample itself, whereas others are caused by the absence of thermal equilibrium between the sample and its environment (e.g., liquid-helium bath). Specific non-equilibrium models based on earlier treatments of the phonon bottleneck, and of spin flips associated with cross relaxation and with level crossings (anticrossings), are discussed. Magnetization data on powders of TMMC diluted with cadmium [i.e., $\left(\mathrm{CH}_{3}\right)_{4} \mathrm{NMn}_{\mathrm{x}} \mathrm{Cd}_{1-\mathrm{x}} \mathrm{Cl}_{3}$, with $0.16 \leq \mathrm{x} \leq 0.50$ ] were measured at $0.55 \mathrm{~K}$ in $18 \mathrm{~T}$ superconducting magnets. The field $B_{1}$ at the first MST from pairs is used to determine the NN exchange constant $J$. This $J / k B$ changes from $-5.9 \mathrm{~K}$ to $-6.5 \mathrm{~K}$ as $\mathrm{x}$ increases from 0.16 to 0.50 . The magnetization curves obtained in the superconducting magnets are compared with simulations based on the equilibrium theory. A reasonably good agreement is found. Data for the differential susceptibility, $d M / d B$, were taken in pulsed magnetic fields (7.4 ms duration) up to $50 \mathrm{~T}$. The powder samples were in direct contact with a $1.5 \mathrm{~K}$ liquid-helium bath. Non-equilibrium effects, which became more severe as $\mathrm{x}$ decreased, were observed. For $\mathrm{x}=0.50$ the non-equilibrium effects are tentatively interpreted using the "Inadequate Heat Flow Scenario," developed earlier in connection with the phonon bottleneck problem. The more severe non-equilibrium effects for $\mathrm{x}=0.16$ and 0.22 are tentatively attributed to cross-relaxation, and to crossings (more accurately, anticrossings) of energy levels, including those of excited states. For $\mathrm{x}=0.16$ (lowest $\mathrm{x}$ ), no MST's were observed above $20 \mathrm{~T}$, which is attributed to a very slow spin relaxation for pairs, compared to a millisecond. A definitive interpretation of this and some other non-equilibrium effects is still lacking.

PACS numbers: $\quad$ 75.50.Ee, 71.70.Gm, 75.10.Jm, 75.60.Ej
\end{abstract}

\section{INTRODUCTION}

Spin clusters with predominantly antiferromagnetic (AF) interactions exhibit steps in the equilibrium magnetization as a function of magnetic field. These magnetization steps (MST's) arise from energy-level crossings which change the ground state. They are observed at very low temperatures when only the ground state contributes to the magnetization $M$. In recent years MST's have yielded a wealth of information about AF clusters, first in diluted magnetic materials and later in molecular magnetism. An overall review of MST's was published recently $\stackrel{1}{*}$ For recent reviews of the magnetic properties of molecular clusters, including MST's, see Ref. 2 .
In a molecular crystal the AF clusters are normally all of one type. The MST's then give values of exchange constants and anisotropy parameters for that cluster type. A diluted magnetic material, on the other hand, contains numerous types of spin clusters. Different cluster types give rise to different series of MST's. In addition to exchange constants and anisotropy parameters, the MST's also give information concerning the populations of the different cluster types. The populations are related to the magnitudes of the MST's in the different series. The results for the cluster populations can be used to check if the distribution of the magnetic ions is random.

Most previous studies of MST's in diluted magnetic crystals were on three-dimensional (3D) materials, $\frac{1}{,}$ although some quantum wells were also studied. $\stackrel{3}{*}$ The 
present paper, however, is devoted to MST's from a diluted AF Heisenberg chain (1D). The material studied is TMMC [chemical formula: $\left(\mathrm{CH}_{3}\right)_{4} \mathrm{NMnCl}_{3}$ ] which was diluted by replacing a large fraction of the Mn atoms by Cd. Powder samples of $\left(\mathrm{CH}_{3}\right)_{4} \mathrm{NMn}_{\mathrm{x}} \mathrm{Cd}_{1-\mathrm{x}} \mathrm{Cl}_{3}$, with $\mathrm{x}$ between 0.16 and 0.5 , were investigated. In these materials the non-magnetic $\mathrm{Cd}^{2+}$ ions break the chains of $\mathrm{Mn}^{2+}$ ions into finite segments.

Pure TMMC is probably the closest approximation to an ideal isotropic (Heisenberg) linear AF chain. For reviews of its magnetic properties, with extensive references to original works, see Refs. 4 5 6 6. The 1D magnetic behavior of this compound is due to the crystallographic structure. It contains chains of $\mathrm{Mn}$ ions which at room temperature are along the $c$-axis of the hexagonal structure. The $\mathrm{Mn}$ ions in each chain are linked by $\mathrm{Cl}$ ions. The space between the combined $\mathrm{Mn}-\mathrm{Cl}_{3}-\mathrm{Mn}$ chains is occupied by tetramethylammonium groups.

The strongest magnetic interaction in TMMC is the isotropic exchange between nearest neighbor (NN) $\mathrm{Mn}^{2+}$ ions in the chain. The NN exchange constant, obtained from various experiments (e.g., Ref. [8), is $J / k_{\mathrm{B}} \cong$ $-6.6 \mathrm{~K}$, where $k_{\mathrm{B}}$ is the Boltzmann constant. Other intrachain exchange constants are believed to be much smaller, and are usually neglected. The exchange interaction between different chains is orders of magnitude smaller than the intrachain interaction, but is responsible for the long-range AF order below the Néel temperature $T_{N}=0.84 \mathrm{~K}$. The anisotropy in TMMC is mainly due to the dipole-dipole interaction. It is of the easy-plane type, and is two orders of magnitude smaller than the dominant exchange interaction $\underline{\underline{9}}$

At room temperature both TMMC and its $\mathrm{Cd}$ ana$\log \left[\left(\mathrm{CH}_{3}\right)_{4} \mathrm{NCdCl}_{3}\right.$, known as TMCC $]$ have isomorphous hexagonal structures (space group $P 6_{3 / m}$ ). Crystallographic phase transitions at lower temperatures result in a lower symmetry, and in small structural differences between TMMC and TMCC. 8.10.11.12 These differences are often assumed to be unimportant, although it is conceivable that even small changes in the crystal structure have some effect on the magnetic behavior, especially on spin relaxation at low temperatures.

Previous investigations of $\left(\mathrm{CH}_{3}\right)_{4} \mathrm{NMn}_{\mathrm{x}} \mathrm{Cd}_{1-\mathrm{x}} \mathrm{Cl}_{3}$ included measurements of the susceptibility and the Néel temperature as a function of $\mathrm{x}$ (Ref. 13). These results were interpreted theoretically both by the original authors, Dupas and Renard, and by Harada et al $l^{14}$ Susceptibility measurements on a related diluted linear AF chain (DMMC:Cd) were obtained and interpreted by Schouten et al $l^{15}$ The authors of both Refs. 13 and 15 noted the difficulties of preparing alloys with uniform $\mathrm{Cd}$ concentrations.

\section{EQUILIBRIUM THEORY}

In this section a theory for the low temperature magnetization $M$ of a diluted AF Heisenberg chain is pre- sented. It is assumed that the spin system is in thermal equilibrium with a constant-temperature heat reservoir. This equilibrium theory is suitable for interpreting the data that were obtained in the slowly-varying magnetic fields ("dc fields") of the superconducting magnets. The additional considerations needed to interpret the results obtained in pulsed fields, of several ms duration, will be discussed in Sec. III

\section{A. The Model}

MST's from finite AF chains were predicted decades ago, ${ }^{16.17}$ but some more recent theoretical results are also useful. The simplest model for MST's in a diluted magnetic material is the single- $J$ cluster model $\underline{\underline{1}}$ It includes only the largest isotropic exchange constant $J$ and the Zeeman energy. Other exchange constants, and all anisotropies, are ignored. This model, with the NN intrachain exchange constant chosen as $J$, is expected to be a good starting point for $\left(\mathrm{CH}_{3}\right)_{4} \mathrm{NMn}_{\mathrm{x}} \mathrm{Cd}_{1-\mathrm{x}} \mathrm{Cl}_{3}$ (hereafter, TMMC:Cd). All cluster models are applicable only when $\mathrm{x}$ is not too high. However, for a diluted magnetic chain (1D) the single- $J$ model it is expected to hold at least up to $\mathrm{x}=0.5$. All the samples in the present study are in this range.

In the single- $J$ model the magnetic clusters are finite chains, each consisting of $n$ coupled spins. These clusters are treated as independent. The total magnetization $M$ is then the sum of the magnetizations of finite chains with different $n$. Let $\mu_{n}$ be the average magnetic moment of a chain with $n$ spins, and let $N_{n}$ be the number of "realizations," per kg, of a chain with $n$ spins. $\left(N_{n}\right.$ may also be called the "population," per kg, of finite chains with $n$ spins). The magnetization per $\mathrm{kg}$ is then

$$
M=\sum N_{n} \mu_{n} .
$$

If $P_{n}$ is the probability that a spin is in a chain with $n$ spins, and if $N_{\text {total }}$ is the total number of spins per $\mathrm{kg}$, then

$$
N_{n}=N_{\text {total }} P_{n} / n \text {. }
$$

The probabilities $P_{n}$ are obtained from well known results, 18

$$
P_{n}=n \mathrm{x}^{n-1}(1-\mathrm{x})^{2}
$$

This result assumes a random distribution of the magnetic ions. This crucial assumption is discussed later. Figure 1 shows the probabilities $P_{n}$ for $n \leq 5$, and the probability $P_{>5}$ that a spin is in a finite chain $n>5$, i.e.,

$$
P_{>5}=1-\sum_{1}^{5} P_{n}=(6-5 \mathrm{x}) \mathrm{x}^{5} .
$$




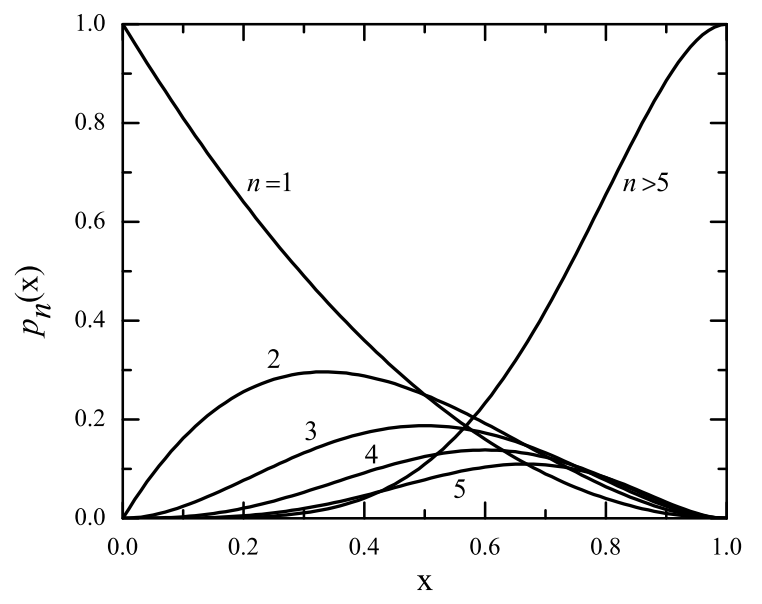

FIG. 1: The probability $P_{n}$ that a magnetic ion is in a finite linear chain with $n$ spins. Results for $n \leq 5$ are plotted as a function of the fraction $\mathrm{x}$ of cations that are magnetic. Also plotted is the probability $P_{>5}$ that a magnetic ion is in a cluster with more than 5 spins.

From Eqs. (13), the magnetization $M$ is given by the infinite sum

$$
M=N_{\text {total }} \sum_{n} \mathrm{x}^{n-1}(1-\mathrm{x})^{2} \mu_{n} .
$$

In practice, exact results for the average magnetic moment $\mu_{n}$ are available only for values of $n$ which are not too large (short chains). The infinite sum in Eq. (5) is therefore truncated at some maximum value of $n$, called $n_{\max }$. Chains with $n>n_{\max }$ are treated using an approximation ${ }^{1}$ In the present work we chose $n_{\max }=5$ because exact results for chains with up to five $\mathrm{Mn}^{2+}$ ions $(S=5 / 2)$ were readily available from previous works $\frac{1.19}{.19}$

When $\mathrm{x}$ is below 0.50 , less than $11 \%$ of the spins are in chains with $n>5$. For this range of $\mathrm{x}$, the "rise and ramp" approximation 1 can be used for the total contribution from chains with $n>5$ [i.e., it approximates the remainder after the sum in Eq. (15) is truncated]. The approximation smoothes the $B$-dependence of this remainder, i.e., MST's from chains with $n>5$ are not resolved. This approach (exact treatment for $n \leq 5$, and an approximation for $n>5$ ) proved to be adequate for interpreting the data obtained in dc magnetic fields. However, the interpretations of some data obtained in pulsed magnetic fields also used available theoretical results 20 for $n>5$.

To implement the rise and ramp approximation, the so-called "short chain model" (Ref. 15) was adopted. This model is appropriate for $\mathrm{x}<0.5$ because more than $99.4 \%$ of the spins are in chains with $n \leq 10$, and more than $99.9 \%$ are in chains with $n \leq 14$. A chain with 14 spins is still short enough to be described by that model. In the short-chain model the ground state at $B=0$ has total spin $S_{T}(0)=0$ when $n$ is even, and $S_{T}(0)=S$ if $n$ is odd. This simple result does not hold for 3D materials, which is one of the reasons why the rise and ramp approximation is simpler and much more accurate for diluted chains than for diluted 3D materials.

\section{B. Qualitative shape of the magnetization curve at low temperatures}

\section{Clusters of one type}

Consider first an ensemble of identical finite chains, all with the same $n$. (In the language of Ref. 1, this is an ensemble of "realizations" of a chain "type" with $n$ spins.) At low temperatures, $k_{B} T \ll|J|$, the qualitative variation of $\mu_{n}$ with $B$ depends on whether $n$ is even or odd. When $n$ is odd the zero-field-ground-state total spin $S_{T}(0)=S$ aligns rapidly at low $B$. This alignment is given by the Brillouin function (BF) for spin $S$. The rapid rise of $\mu_{n}$ at low $B$ ends when the BF approaches saturation. After the BF saturates, there is a magnetic-field interval in which $\mu_{n}$ is nearly constant. At still higher $B$ a series of MST's appears. The fields at these MST's depend on $n$. Once this series of MST's is completed, $\mu_{n}$ reaches its true saturation value $\mu_{n, \max }=n g \mu_{\mathrm{B}} S$.

When $n$ is even, $S_{T}(0)=0$. Therefore, no initial fast rise of $\mu_{n}$ occurs at low $B$, in contrast to the case of odd $n$. However, at high magnetic fields a series of MST's still appears. At the completion of this series, $\mu_{n}$ reaches its true saturation value.

\section{Total magnetization $M$}

Chains with all values of $n$ contribute to the total magnetization $M$. When $k_{B} T \ll|J|$, the chains with odd values of $n$ produce a fast rise of $M$ at low $B$. This rise follows the BF for spin $S$. After this fast rise is completed, and before the appearance of the first MST of significant size, there exists a field interval in which $M$ stays approximately constant. This (nearly) constant value, $M_{s}$, is the "apparent saturation value" (see Ref. 1). At still higher fields, MST's series from chains with $n \geq 2$ appear. Once the last MST of significant size is completed, $M$ reaches its true saturation value $M_{0}$. As discussed later, the magnetic field required to saturate the magnetization of a chain remains finite even when $n \rightarrow \infty$, so that $M$ reaches true saturation at a finite $B$.

\section{Apparent saturation value}

The true saturation value of the magnetization is

$$
M_{0}=N_{\text {total }} g \mu_{\mathrm{B}} S .
$$

The apparent saturation value is

$$
M_{s}=\sum_{n=\text { odd }} N_{\text {total }} \mathrm{x}^{n-1}(1-\mathrm{x})^{2} g \mu_{\mathrm{B}} S,
$$


where the sum is only over odd $n$. Therefore,

$$
M_{s} / M_{0}=\sum_{n=\mathrm{odd}} \mathrm{x}^{n-1}(1-\mathrm{x})^{2} .
$$

This infinite geometric series can be summed, $\stackrel{21}{,}$

$$
M_{s} / M_{0}=(1-\mathrm{x}) /(1+\mathrm{x}) .
$$

It is noteworthy that an exact analytical expression for $M_{s}$ was not obtained for 3D materials $\frac{1}{\underline{\underline{1}}}$ A rough approximation was then used for the net contribution from clusters with $n>n_{\max }$. This approximation was used only for $\mathrm{x} \lesssim 0.1$. In contrast, Eq. (9) for a diluted chain is exact, within the framework of the short-chain model. It should be very accurate for $\mathrm{x} \leq 0.5$.

\section{MST's from Chains with $n \leq 5$}

All chains with $n \geq 2$ give rise to MST's. The magnetic fields $B_{i}$ at the MST's from chains composed of spins $S=5 / 2$ were given earlier for $n=2$ (pairs, or dimers), $n=3$ (triplets, or trimers), $n=4$ (quartets, or tetramers), and $n=5$ (quintets, or pentamers) $\frac{19}{n}$ For completeness, the values of the reduced fields $b_{i}=g \mu_{\mathrm{B}} B_{i} /|J|$ are repeated here.

For $n=2$ there are five MST's at $b_{i}=2,4,6,8,10$. For $n=3$ there are five MST's are at $b_{i}=7,9,11,13,15$. For $n=4$ there are ten MST's at $b_{i}=0.95,2.04,3.39,5.02$, $6.87,8.85,10.88,12.94,15.00,17.07$. For $n=5$ there are ten MST's at $b_{i}=4.62,5.89,7.18,8.49,9.86,11.29,12.83$, 14.48, 16.24, 18.09 .

Figure 2 shows the zero-temperature values of $\mu_{n}$ as a function of $b$ for $n=1,2,3,4,5$. At finite $T$ the ground state of a chain is not the only contributor to $\mu_{n}$. It is then necessary to include all energy levels in the calculations of $\mu_{n}$. The procedures for such calculations were discussed in Ref. 1 .

\section{E. Rise and ramp approximation for chains with $n>5$}

The total contribution of chains with $n>5$ to $M$ is approximated by a sum of two terms: 1) a fast rise at low $B$, which follows the $\mathrm{BF}$ for spin $S$, and 2) a linear "ramp" from $B=0$ up to an effective saturation field $B_{s}$.

The fast rise at low $B$ is due to chains with odd $n$, starting with $n=7$. Its magnitude is

$$
(\Delta M)_{\text {rise }}=M_{0} \mathrm{x}^{6}(1-\mathrm{x}) /(1+\mathrm{x}) .
$$

The linear ramp approximates the superposition of numerous MST's from all chains with $n>5$. The ramp is given by

$$
M_{\mathrm{ramp}}=\left\{\begin{array}{l}
(\Delta M)_{\mathrm{ramp}}\left(B / B_{s}\right), \text { for } B \leq B_{s} \\
(\Delta M)_{\mathrm{ramp}}, \quad \text { for } B>B_{s}
\end{array}\right.
$$

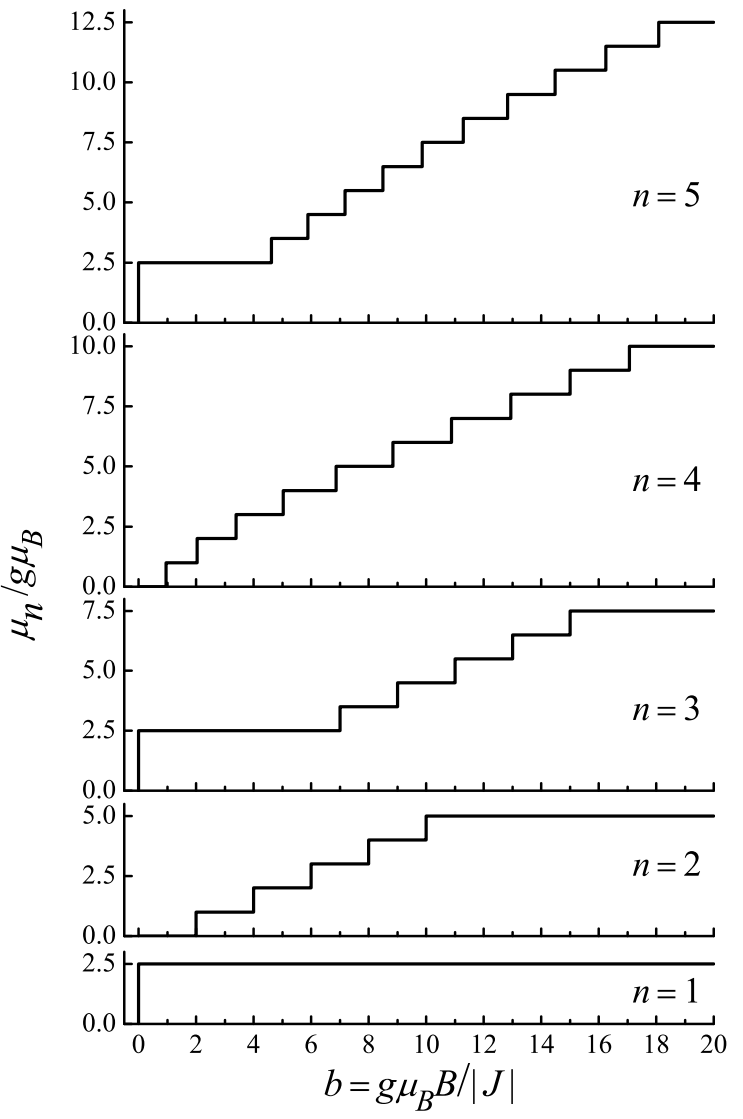

FIG. 2: Magnetic moment per cluster, $\mu_{n}$, for chains with $n \leq 5$ at $T=0$, plotted as a function of the reduced field $b=g \mu_{\mathrm{B}} B /|J|$.

where

$$
\begin{aligned}
(\Delta M)_{\mathrm{ramp}} & =P_{>5} M_{0}-(\Delta M)_{\text {rise }} \\
& =M_{0} \mathrm{x}^{5}\left(6-4 \mathrm{x}^{2}\right) /(1+\mathrm{x}) .
\end{aligned}
$$

The reduced field $b_{s}(n)$ where the magnetization of a finite chain with $n$ spins reaches saturation at $T=0$ increases with $n$. However, in the limit $n \rightarrow \infty$ it is still finite $\stackrel{17.19}{=}$ namely, $b_{s}(n=\infty)=8 S$. It can be shown that the $n$-dependence of $b_{s}(n)$ is given by the equation

$$
b_{s}(n)=8 S \cos ^{2}(\pi / 2 n) .
$$

For chains with $n>5$ the change of this function is only $7 \%$. In the present case of $S=5 / 2, b_{s}(n)$ changes from 18.7 to 20.0 when $n$ increases from 6 to $\infty$. The value $b_{s}=19$, corresponding to

$$
g \mu_{\mathrm{B}} B_{s}=19|J|,
$$

will be used in Eqs. (11) for the ramp.

Figure 3(a) shows the predicted zero-temperature magnetization curve for $S=5 / 2$ when $\mathrm{x}=0.50$. Figure 3 (b) is an expanded view for the range of magnetic fields relevant to the present experiments. The integers in this 

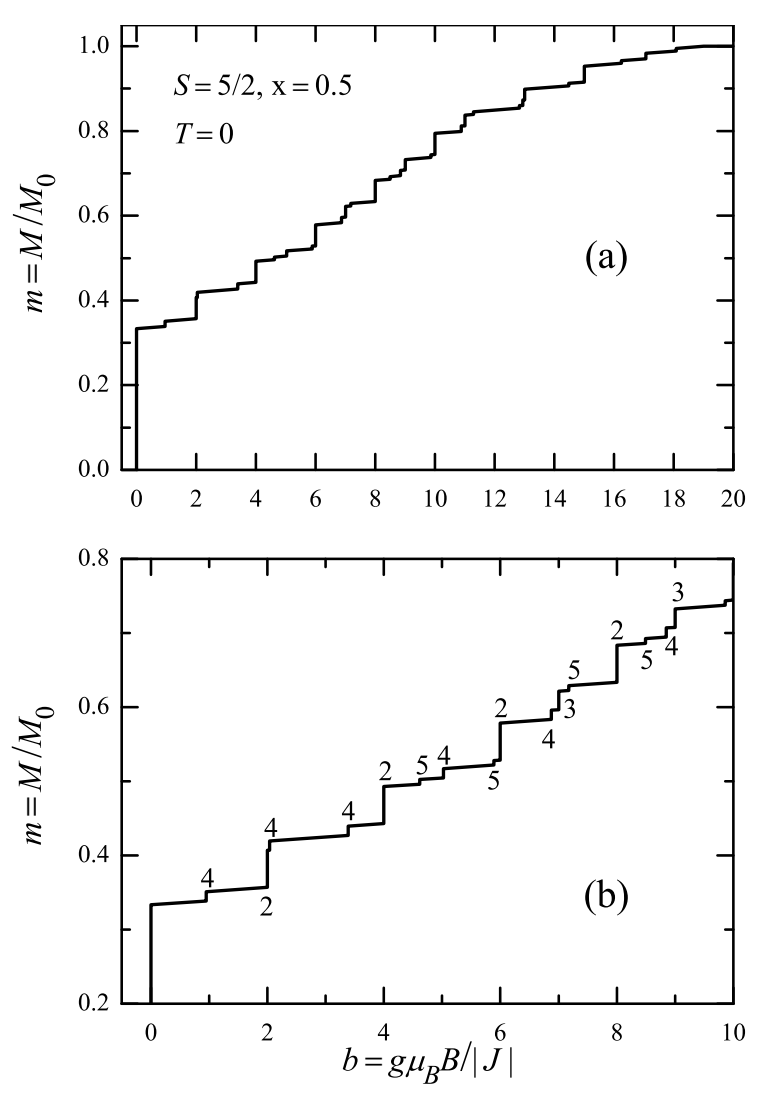

FIG. 3: a) Calculated zero-temperature equilibrium magnetization of a diluted linear chain with $\mathrm{x}=0.5$ as a function of the reduced field $b$. These results are for $S=5 / 2$. b) Expanded view of part (a) for the range of reduced fields relevant to the present work. The values of $n$ for the chains responsible for each MST are indicated.

figure are the values of $n$ for the finite chains responsible for each MST.

\section{F. Random Distribution}

Some of the preceding results used the probabilities $P_{n}$ for a random distribution of the magnetic ions. Although a random distribution is often found in diluted magnetic materials, non-random distributions were also observed $\stackrel{1}{\underline{1}}$ In the case of TMMC:Cd, difficulties of obtaining a uniform Mn distribution were reported in the literature 13.15 Therefore, the possibility of a non-random distribution cannot be ignored. The effects on the magnetization curve caused by departures from a random distribution were discussed in Ref. 1. For example, a tendency of the magnetic ions to cluster together is expected to decrease $M_{s}$.

\section{NON-EQUILIBRIUM EFFECTS}

\section{A. Non-Equilibrium Effects}

Experiments are normally conducted with the sample in contact with, or near, a thermal reservoir of constant temperature, e.g., a liquid helium bath at a temperature $T_{\text {bath }}$. In some experiments the sample is not in thermal equilibrium either within itself and/or with the thermal reservoir. That is, the time for reaching complete equilibrium (both internal and with the reservoir) is not short compared to the time of the experiment. Such non-equilibrium cases require special considerations.

Thermal equilibrium is usually maintained if 1 ) the magnetic field $B$ is swept slowly ("dc fields"), and 2) the sample is in good thermal contact with the reservoir. These conditions are often fulfilled in superconducting magnets when the sample is immersed in liquid helium. There are, however, exceptional cases of non-equilibrium behavior even for slowly varying $B$ and good thermal contact. These have been discussed extensively in connection with macroscopic quantum tunnelling. A well known example is $\mathrm{Mn}_{12}$-acetate 2.22 .23 .24 .25

Departures from thermal equilibrium with the reservoir due to imperfect thermal contact were observed and discussed for a wide range of sweep rates, $d B / d t$, from typical sweep rates in "dc magnets" to the very fast rates in pulsed magnets $26.27 .28 .29 .30,31.32 .33 .34$ The extreme case of a sample isolated from the thermal reservoir (adiabatic conditions) was discussed by Wolf long ago, assuming thermal equilibrium within the sample 35 The magnetocaloric effect leads to cooling when any one of the MST's is approached. Such "cooling by adiabatic magnetization" has been observed many times in both dc fields and pulsed fields ${ }^{26,29,32,33.36}$

\section{B. Some non-equilibrium models for pulsed magnetic fields}

\section{Classification of non-equilibrium behaviors}

Non-equilibrium behavior in pulsed fields of milliseconds duration is relevant to the interpretation of the present pulsed field data. For this purpose it will be useful to distinguish between three types of non-equilibrium situations:

1. The spin-lattice relaxation is fast enough so that thermal equilibrium within the sample is established in a time which is very short compared to the pulse duration. The non-equilibrium behavior is then due to an inadequate heat flow between the liquid-helium bath and the sample, i.e., the samplebath equilibration time is not short compared to the pulse duration. The "Inadequate Heat Flow" models are appropriate for this situation. 
2. The time for establishing thermal equilibrium within the sample is not short compared to the pulse duration, but there is adequate sample-tobath heat flow. The non-equilibrium is then governed by the slow spin-lattice relaxation processes. There are many such processes. Here, only spinflips associated with level crossings or with crossrelaxation $(\mathrm{CR})$ will be discussed 37.38

3. Both the time for reaching equilibrium within the sample, and the time for reaching a sample-to-bath equilibrium are not short compared to the pulse duration. Some such cases were considered in the literature $\underline{30.31 .33 .39}$

\section{Inadequate Heat Flow (IHF) Models}

The phonon bottleneck (PB) phenomenon has been known for a long time ${ }^{40.41}$ Treatments of this phenomenon in the context of the magnetization process of magnetic clusters led to the development of several IHF models 27.29 .30 .31 .33 .34 The common feature of these models is that the spin and phonon subsystems, within the sample, are very nearly in thermal equilibrium. The spin temperature $T_{s}$, the phonon temperature, and the sample temperature are the same. However, the sample and the helium bath are not in thermal equilibrium $\left(T_{s} \neq T_{\text {bath }}\right)$. Different IHF models treat the sample-to-bath heat flow differently. For a solid sample in contact with a liquidhelium bath the heat flow was assumed to be limited by the Kapitza resistance. ${ }^{27.29 .34}$ Many of the qualitative features of the magnetization curve are common to all IHF models; they do not depend on the detailed treatment of the heat flow.

Because the sample is internally in equilibrium, the equilibrium theory in Sec. III still applies. The absence of equilibrium with the helium bath enters only in the time dependence (and, hence, the $B$-dependence) of the spin temperature $T_{s}$. This dependence has been calculated using various IHF models 27.29.30.31.33 Approaching the energy-level crossing (strictly, anticrossing) associated with each MST, $T_{s}$ decreases. After passing through the anticrossing region, $T_{s}$ increases. If some heat flows between the sample and the bath then the latter increase is large enough that $T_{s}$ is temporarily above $T_{\text {bath }}$. Miyashita and co-workers ${ }^{31}$ have called this behavior of $T_{s}$ "the magnetic Foehn effect."

The $B$-dependence $T_{s}$ can lead to the following qualitative effects: 1) The $d M / d B$ peaks are narrower than the thermal width. 2) The $d M / d B$ peaks are asymmetric, i.e., the rise of $d M / d B$ as the MST is approached is faster than the fall after passing through the MST. This is true both for increasing and decreasing $B$. 3) The magnetization and $d M / d B$ exhibit hysteresis. 4) Under some conditions, a small "satellite" MST appears after the main MST. All these effects have been observed experimentally.

\section{Cross Relaxation (CR) Model}

A severe non-equilibrium behavior, not explainable by IHF models, was observed in pulsed field experiments by Ajiro et al . $^{37}$ It was interpreted in terms of CR between pairs and singles, and also between the pairs themselves. More recently, CR was discussed in the context of tunneling theory ${ }^{38,39} \mathrm{CR}$ can involve both ground and excited states.

$\mathrm{CR}$ is one of the mechanisms of spin relaxation. It involves simultaneous spin flips in weakly coupled clusters. The model of Ajiro et al. also includes a single spin flip in only one of the clusters. The later is actually not a CR process, and is better described as tunneling. In this model the spin relaxation rate is appreciable only at some values of $B$.

The simplified picture used in Ref. 37 ignored small level repulsions near level crossings. (Level repulsion is included in more detailed models that are based on tunneling, 38.39 ) In this simplified picture the relaxation rate can be fast only if simultaneous CR spin flips, or a single spin flip, do not change the total energy of the spin system. For a single spin flip, in one cluster, this happens at energy-level crossings for this cluster. These level crossings include those of excited states. For CR between two weakly coupled clusters the energy-level separation in one cluster should match a level separation in the other. The two clusters may be of the same type (e.g., two coupled pairs) or of different types (e.g., a pair and a single). A similar criterion for energy-level separations applies to CR between three, or more, coupled clusters.

In the equilibrium theory, the $d M / d B$ peaks associated with MST's from $\mathrm{Mn}^{2+}$ pairs occur at fields

$$
g \mu_{\mathrm{B}} B_{i}=2 i|J|,
$$

where $i=1,2, \ldots,(2 S+1)$. In the model of Ajiro et al. these peaks from pairs are called the "fundamental" peaks, and they are labeled as $\mathrm{P}_{i}$. Specializing to pairs composed of $\mathrm{Mn}^{2+}$ ions, there are five such peaks. In addition to the fundamental peaks, other peaks are also predicted. The most pronounced are the "second harmonic" peaks $\mathrm{P}_{m / 2}$ at fields $B_{m}=m\left(B_{1} / 2\right)$, with $m=1$ to 10 . The five peaks for odd $m$ were observed clearly by Ajiro et al. The peaks for even $m$ coincide with the fundamental peaks.

In addition to the second-harmonic peaks, fifteen third harmonic peaks $\mathrm{P}_{k / 3}$ at $B_{k}=k\left(B_{1} / 3\right)$, were predicted. Many of these were also observed. Fourth and sixth harmonic peaks were also discussed. These results are from a model which involves only singles and pairs. The model can be extended to include other clusters.

Some of the simultaneous spin-flip transitions which can give rise to $\mathrm{P}_{1 / 2}$ (the first of the ten second-harmonic peaks) are shown in Figs. 4(a) and 4(b). Note that in either case, each of the two simultaneous spin flips increase the component of the spin along $B$ by one unit. Figure 5 shows a few level crossings that may contribute to various peaks: the first three fundamental peaks $\mathrm{P}_{1}, \mathrm{P}_{2}$, 


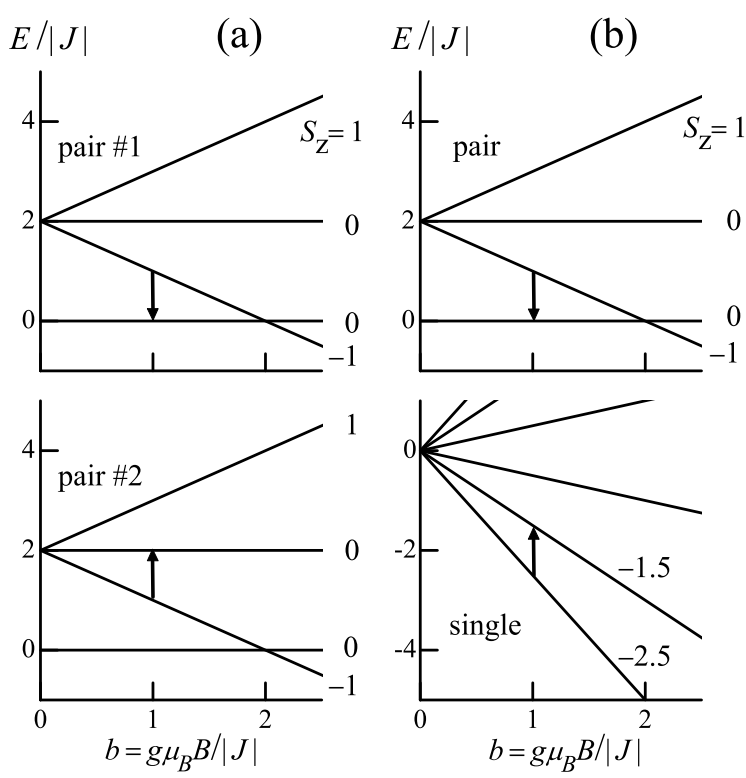

FIG. 4: Some CR spin-flip transitions which may lead to the $\mathrm{P}_{1 / 2}$ peak in $d M / d B$. This peak is at $B=|J| / g \mu_{\mathrm{B}}$. a) Simultaneous spin flips in two pairs. b) Simultaneous spin flips in a pair and in a single.

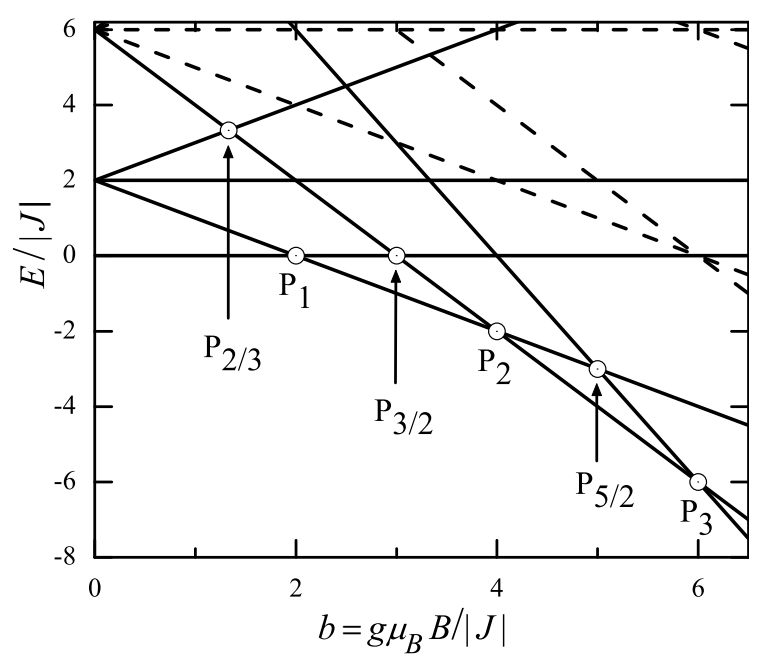

FIG. 5: Some energy-level crossings which may contribute to the "fundamental" peaks $\mathrm{P}_{1}, \mathrm{P}_{2}$, and $\mathrm{P}_{3}$, and to the "harmonic" peaks $\mathrm{P}_{3 / 2}, \mathrm{P}_{5 / 2}$, and $\mathrm{P}_{2 / 3}$. The $\mathrm{P}_{k / r}$ peak is the $k$-th peak of the $r$-th harmonic in the model of Ajiro et al ${ }^{37}$

$\mathrm{P}_{3}$; the third and fifth second-harmonic peaks, $\mathrm{P}_{3 / 2}$ and $\mathrm{P}_{5 / 2}$; and the second third-harmonic peak, $\mathrm{P}_{2 / 3}$. Cross relaxation processes may also contribute to some of the same peaks, e.g., to $\mathrm{P}_{2 / 3}$.

\section{EXPERIMENTAL TECHNIQUES}

\section{A. Sample preparation}

The procedure of growing crystals of TMMC:Cd followed Ref. 13. The samples were grown by evaporation from water solutions of $\mathrm{MnCl}_{2} \cdot 4 \mathrm{H}_{2} \mathrm{O},\left(\mathrm{CH}_{3}\right)_{4} \mathrm{NCl}$, $\mathrm{CdCl}_{2} \cdot 2 \mathrm{H}_{2} \mathrm{O}$, and some $\mathrm{HCl}$ acid. The solutions were maintained at $30^{\circ} \mathrm{C}$. As already noted, the Cd concentration in the crystallized samples is about 50 times larger than in the starting solution ${ }^{13.15}$ Because the Cd concentration in the solution decreases rapidly as the crystal growth progresses, a large solution volume $(300 \mathrm{ml})$ was used to crystalize a "product" of TMMC:Cd with a total mass of about $100 \mathrm{mg}$. The large starting volume increased the uniformity of the $\mathrm{Cd}$ concentration in the product.

Physically, the product consisted of many needleshaped crystals. The long dimension of a needle (up to $4 \mathrm{~mm}$ ) was parallel to the hexagonal axis. The color gradually changed from pink towards white as the Mn concentration $\mathrm{x}$ decreased. Each of the magnetization measurements, in both dc magnetic fields and in pulsed fields, used only a portion of the product, typically $30 \mathrm{mg}$.

The $\mathrm{Mn}$ concentration $\mathrm{x}$, was determined from the high-temperature dc susceptibility, $\chi=M / B$, measured using a SQUID magnetometer. The magnetic field $B=$ $0.1 \mathrm{~T}$ was well within the range where $\chi$ is independent of $B$. In the temperature range from $150 \mathrm{~K}$ to $300 \mathrm{~K}$, the data for $\chi$ were well described by a sum of a Curie-Weiss susceptibility and a constant due to the diamagnetism of the lattice. The concentration $\mathrm{x}$ was obtained from the Curie constant $C$. Strictly, the Curie-Weiss law is accurate only in the limit of very high temperatures. The percentage error in the Curie constant, resulting from the use of data between $150 \mathrm{~K}$ and $300 \mathrm{~K}$, depends on the Mn concentration. Based on the results in Ref. 42, for the samples used here the error in $C$, and hence in $\mathrm{x}$, was less $2 \%$. The percentage error in the Curie-Weiss temperature $\theta$ is larger than for $C$, but $\theta$ does not enter into the determination of $\mathrm{x}$.

Values of $\mathrm{x}$ for several portions of the $\sim 100 \mathrm{mg}$ product obtained from a single solution were close to each other. A quantitative comparison between experimental dc magnetization data and theoretical simulations was carried only for samples 2, 4, and 5 in Table【 To increase the confidence in this comparison, values of $\mathrm{x}$ were determined for the very same three samples. As a check, the $\mathrm{Mn}$ and $\mathrm{Cd}$ weight percents for these three samples were also determined directly by atomic emission spectroscopy with inductively coupled plasma (ICP-AES). The values of $\mathrm{x}$ deduced from ICP-AES were in reasonable agreement with the values from the susceptibility (see Table【).

The magnetization of several other samples $\left(1^{*}, 3^{*}, 4^{*}\right.$, and $5^{*}$ in Table 【) was also measured in dc fields. However, for these samples each value of $\mathrm{x}$ is from susceptibility data on a different portion of the same product. Samples 4 and $4^{*}$ are two different portions of the same 
TABLE I: Properties of the various samples. The Mn concentration $\mathrm{x}$, as determined from the magnetic susceptibility (Suscept.) and from atomic emission spectroscopy with inductively coupled plasma (ICP-AES). The magnetic field $B_{1}$ is at the first MST from pairs, and the magnetic field $B_{1 \mathrm{QUART}}$ is at the first MST from quartets. Both fields were determined from dc magnetization data. The NN exchange constant $J$ was obtained from $B_{1}$.

\begin{tabular}{lccccc}
\hline \hline $\begin{array}{l}\text { Sample } \\
\text { No. }\end{array}$ & $\begin{array}{c}\mathrm{x} \\
\text { Suscept. }\end{array}$ & $\begin{array}{c}\mathrm{x} \\
\text { ICP-AES }\end{array}$ & $\begin{array}{c}B_{1} \\
(\mathrm{~T})\end{array}$ & $\begin{array}{c}J / k_{\mathrm{B}} \\
(\mathrm{K})\end{array}$ & $\begin{array}{c}B_{\text {1QUART }} \\
(\mathrm{T})\end{array}$ \\
\hline $1^{*}$ & 0.16 & & 8.85 & -5.94 & \\
\hline 2 & 0.22 & 0.22 & 8.70 & -5.85 & 3.8 \\
$2^{*}$ & 0.22 & & & & \\
\hline $3^{*}$ & 0.27 & & 8.85 & -5.94 & \\
\hline 4 & 0.25 & 0.30 & 9.10 & -6.11 & 4.3 \\
$4^{*}$ & 0.28 & & 8.90 & -5.99 & 4.5 \\
\hline 5 & 0.48 & 0.50 & 9.65 & -6.48 & 4.4 \\
$5^{*}$ & 0.50 & & 9.65 & -6.48 & 4.4 \\
\hline \hline
\end{tabular}

product, as are samples 5 and $5^{*}$.

$\mathrm{X}$-ray powder diffraction patterns were obtained at room temperature using $\mathrm{Cu}-\mathrm{K}_{\alpha}$ radiation. Data were taken on two samples from the same products as those of samples $1^{*}$ and 4 (or $4^{*}$ ). The diffraction patterns for both samples were very similar to the pattern obtained, with the same equipment, on pure $\operatorname{TMCC}(\mathrm{x}=0)$. No additional, or missing, diffraction peaks were observed. These results are consistent with a single crystallographic phase.

\section{B. Magnetization in dc magnetic fields}

Magnetization data in slowly varying magnetic fields (so-called "dc fields") were taken with a vibrating sample magnetometer (VSM). The VSM operated in $18 \mathrm{~T}$ superconducting magnets. The sample was in direct contact with a liquid ${ }^{3} \mathrm{He}$ bath, which was in an insert dewar. The temperature $0.55 \mathrm{~K}$ was reached by pumping on the ${ }^{3} \mathrm{He}$ bath. The field-sweep time (zero to $18 \mathrm{~T}$ ) was about 1 hour.

\section{Differential Susceptibility in pulsed magnetic fields}

The differential susceptibility, $d M / d B$, was measured in pulsed magnetic fields up to $50 \mathrm{~T}(500 \mathrm{kG})$. The techniques have been described earlier ${ }^{29}$ The shape of the field pulse ( $B$ versus time) was approximately a half cycle of a weakly damped sine wave, with a rise time of $3.1 \mathrm{~ms}$, and fall time of $4.3 \mathrm{~ms}$. For each sample, data were first taken with the sample in the pickup coils, and shortly thereafter with the sample outside the pickup coils. The signal from the sample was obtained by taking the difference.
The powder samples used in the pulse field experiments were obtained by crushing the mm-size needles of the growth products. Each sample, consisting of 20 to $30 \mathrm{mg}$ fine powder (grain size less than $0.1 \mathrm{~mm}$ ), was placed in a thin-walled $(0.25 \mathrm{~mm})$ cylindrical capsule made of Delrin. The capsule was immersed in a liquid ${ }^{4} \mathrm{He}$ bath which was maintained at $1.5 \mathrm{~K}$. The Delrin capsule had a small hole at its bottom. The hole (covered by a tissue paper) allowed a direct contact between the sample and the bath of superfluid helium. However, previous experiments have indicated that despite such a direct contact the sample may not be in thermal equilibrium with the bath during the $7.4 \mathrm{~ms}$ field pulse 29.32

\section{MAGNETIZATION IN DC MAGNETIC FIELDS}

\section{A. Experimental Results}

\section{Gross Features}

Figure 6(a) shows magnetization data at $0.55 \mathrm{~K}$ for samples 2,4 , and 5 . These "dc data" were actually obtained with a sweep rate of $\sim 0.3 \mathrm{~T} / \mathrm{min}$. No hysteresis was observed. The (very small) corrections for lattice diamagnetism and addenda are included in Fig. 6] so that the magnetization $M$ is that of the $\mathrm{Mn}^{2+}$ ions. The numerical derivatives, $d M / d B$, of these curves are shown in Fig. 6(b). The Mn concentrations x, measured on the very same samples, are given in Table प

The main features in Fig. 66 (a) are: 1) a fast rise of $M$ at low H, 2) a large MST near 9 or $10 \mathrm{~T}$, and 3) a smaller MST near $4 \mathrm{~T}$. There are also indications of other MST's at higher fields. For example, sample 2, for which the data extend to slightly higher fields than for the other samples, shows the beginning of a large MST near the top of the field. These main features of the dc magnetization curves agree with theoretical predictions for the equilibrium magnetization, such as those in Fig. 3(b). The range of the reduced field $b$ that corresponds to the experimental data in Fig. [6] extends up to about 4 . The exact maximum value of $b$ is slightly different for different curves in this figure.

The fast rise of the $M$ at low $B$ corresponds to the alignment of the zero-field-ground-state spin, $S_{T}(0)$, of finite chains with odd $n$. The main contribution to this fast rise is from the singles $(n=1)$. The large MST observed near 9 or $10 \mathrm{~T}$ corresponds, essentially, to the first MST from pairs (clusters with $n=2$ ). Other contributions to this observed MST are from some longer chains that have a MST at nearly the same field. For example, the second MST from quartets (chains with $n=4$ ) is predicted to occur at a field which is only $2 \%$ higher than that of the first MST from pairs. Neither the triplets $(n=3)$ nor the quintets $(n=5)$ have a MST near this field. The total contribution of chains with $n>2$ to the observed MST near 9 or $10 \mathrm{~T}$ is expected to be smaller than the contri- 

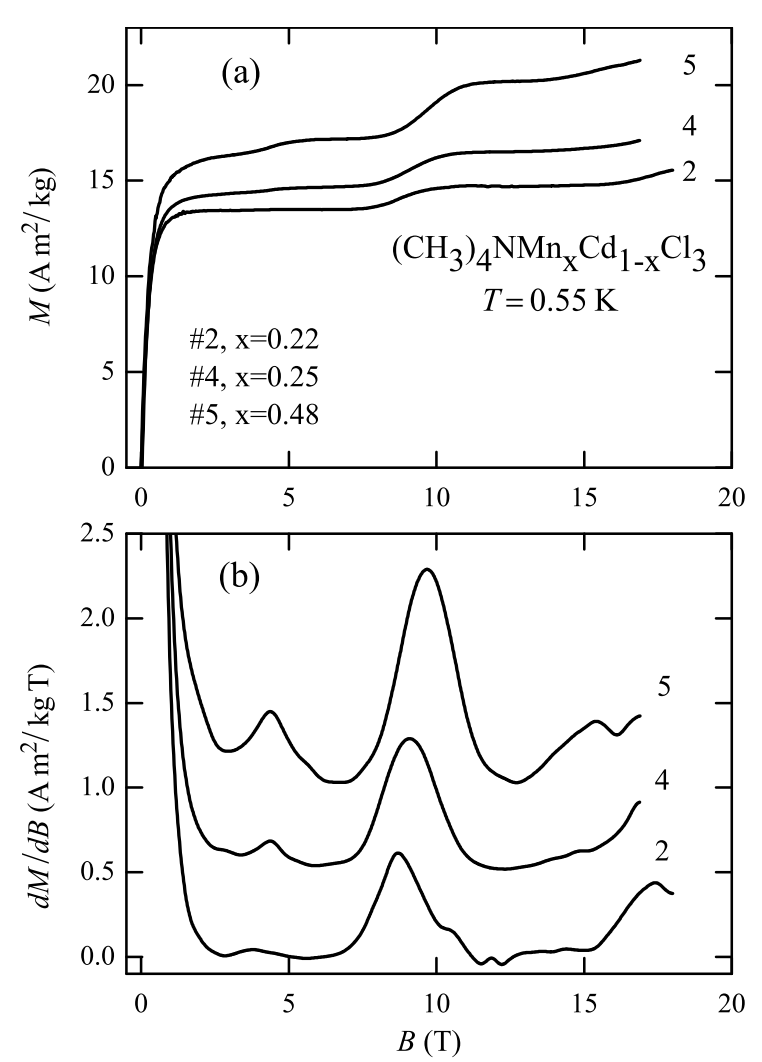

FIG. 6: (a) Magnetization $M$ of samples $2(\mathrm{x}=0.22), 4$ $(\mathrm{x}=0.25)$, and $5(\mathrm{x}=0.48)$, measured at $0.55 \mathrm{~K}$ in dc magnetic fields. The results have been corrected for lattice diamagnetism and addenda. The SI unit $\mathrm{Am}^{2} / \mathrm{kg}$ is equivalent to $1 \mathrm{emu} / \mathrm{g}$. (b) The numerical derivative, $d M / d B$ of the magnetization traces. The derivative curves for samples 4 and 5 have been shifted upwards.

bution from the pairs. The reason is that for $\mathrm{x}=0.5$ the populations $N_{n}$ of these longer chains are small compared to the population $N_{2}$ of the pairs.

For sample 2, a substantial portion of the second MST from pairs is also seen at the highest fields. The peak in the derivative $d M / d B$ for this sample, near the top of the field in Fig. G(b), is close to the expected field for this MST, i.e., $B_{2}=2 B_{1}=17.4 \mathrm{~T}$. The beginning of the second MST from pairs is also seen in the derivative curve for sample 4.

The relatively small MST near $4 \mathrm{~T}$, seen in Figs. 6(a) and (b), is identified as the first MST from the chains with $n=4$ (so-called "string quartets"). The first MST from the quartets is predicted to occur at $B=0.475 B_{1}$, where $B_{1}$ is the field at the first MST from pairs. The experimental results in Table \ are in reasonable agreement with this prediction. The second MST from quartets which, as already noted, is predicted to occur at a field which is only $2 \%$ higher than $B_{1}$, was not resolved at $0.55 \mathrm{~K}$. This was expected because at $0.55 \mathrm{~K}$ the broadening of any MST due to the finite temperature ("thermal broadening") is more than $10 \%$ of $B_{1}$. The third MST from the quartets is also predicted to occur within the field range of Fig. 6] However, the predictions for this MST were not fully confirmed by the data. For example, in Fig. G(b) the derivative curve for sample 5 exhibits a small peak near $15 \mathrm{~T}$. For the same sample, the predicted field at the third MST from the quartets is higher by about $1 \mathrm{~T}$.

Some features of the experimental results in Fig. 6] depend on the Mn concentration $\mathrm{x}$. The first MST from the quartets stands out more clearly as $\mathrm{x}$ increases. This trend is expected from the probability curves in Fig. 1 The cluster populations $N_{n}$ are related to these probabilities by Eq. (21). As $x$ increases there is an increase in population ratios $N_{4} / N_{2}$ between quartets and pairs, and $N_{4} / N_{1}$ between quartets and singles. Therefore, as $\mathrm{x}$ increases, the MST from quartets stands out more clearly in comparison with the MST from pairs, and also in comparison with fast magnetization rise at low fields.

Another feature that depends on $\mathrm{x}$ is the value of the magnetic field at the large MST near 9 or $10 \mathrm{~T}$. In Fig. 6(b) the peak associated with this MST shifts to slightly higher fields as $\mathrm{x}$ increases. The field at this peak is expected to be very close to $B_{1}$. Numerical values as a function of $\mathrm{x}$ are listed in Table 1 The change of $B_{1}$ is attributed to a slight dependence of the NN exchange constant $J$ on the Mn concentration.

\section{2. $N N$ Exchange Constant}

The NN intrachain exchange constant $J$ was obtained from the field $B_{1}$ of the first MST from pairs, using Eq. (15) and assuming $g=2.00$ for the $\mathrm{Mn}^{2+}$ ion. Values of $J$ for all the samples are given in Table प The exchange constant for $\mathrm{x}=0.5, J / k_{\mathrm{B}}=-6.5 \mathrm{~K}$, is about $10 \%$ higher than for $\mathrm{x}=0.22$. This $10 \%$ change is too large to be accounted for by the unresolved MST from the quartets. As already mentioned, $J / k_{\mathrm{B}} \cong-6.6 \mathrm{~K}$ for pure TMMC $(\mathrm{x}=1)$. We are not aware of any theoretical calculation of the x-dependence of $J$ in this system.

\section{B. Comparison with Simulations}

Figures 7 9 compare the experimental results with numerical simulations based on equilibrium theory for the single- $J$ model (Section III). The comparison is for the normalized magnetization $m=M / M_{0}$, where $M_{0}$ is the true saturation magnetization. The "experimental" curves use the measured $M$ (Fig. 6) and the calculated saturation magnetization $M_{0}$ for the Mn concentration $\mathrm{x}$. The simulations assume a random distribution of the Mn ions, and use the values of $J$ and $\mathrm{x}$ (Suscept.) given in Table $\llbracket$ for that sample. Clusters with $n \leq 5$ are treated exactly, and the rise-and-ramp approximation is used for the total contribution of larger clusters. There are no adjustable parameters in the simulations. 


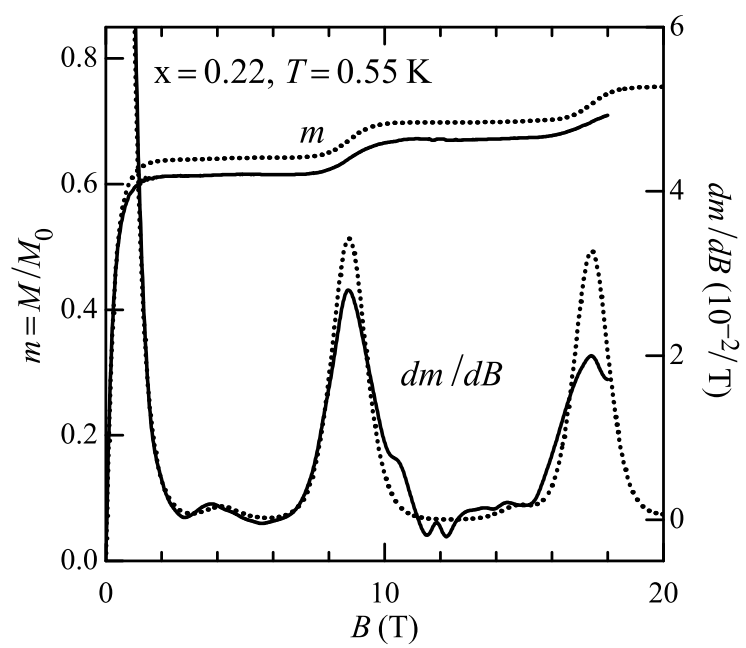

FIG. 7: Comparison between the dc data for $\mathrm{x}=0.22$ at $0.55 \mathrm{~K}$ with a simulation based on equilibrium theory for the single- $J$ model. The left ordinate scale is for the normalized magnetization $m=M / M_{0}$, where $M_{0}$ is the true saturation value. The right ordinate scale is for $d m / d B$. Solid curves are from the data in Fig. 6 and the calculated $M_{0}$. The dotted curves are from the simulation. Only thermal broadening, at the actual temperature $0.55 \mathrm{~K}$, is included in the simulation.

For sample 2, with $\mathrm{x}=0.22$, the agreement between experiment and theory is reasonably good (Fig. [7). The difference between the measured and simulated magnetizations is a few percent. It is comparable to the total experimental uncertainty, mainly from the uncertainty in $x$. The observed magnitude of the first MST from the pairs is in agreement with the simulation. However, the associated experimental $d M / d B$ peak is somewhat broader than in the simulation. Physical mechanisms that broaden MST's were discussed in Ref. 11. In the present case thermal broadening at the experimental temperature, $T=0.55 \mathrm{~K}$, is expected to be the strongest of these mechanisms. It is the only broadening mechanism that was included in the simulations. Non-thermal causes of line broadening include the dipole-dipole interaction and local strains associated with the random replacement of Mn by Cd. Because non-thermal broadening was neglected in the simulation, it is not surprising that the experimental $d M / d B$ peaks are somewhat broader. The numerical differentiation of $M$ with respect to $B$ also broadens the experimental peak slightly.

Figure 8 shows that for $\mathrm{x}=0.25$ the agreement between experiment and simulation is, again, reasonably good. However, the observed $d M / d B$ peak at $4.3 \mathrm{~T}$, from the first MST for quartets, is somewhat larger than expected. The simplest interpretation is that the number of quartets is larger than given by a random distribution. The behavior of the derivative $d m / d B$ near $15 \mathrm{~T}$ is attributed to the third MST from quartets.

Figure 9] shows that the agreement between experiment

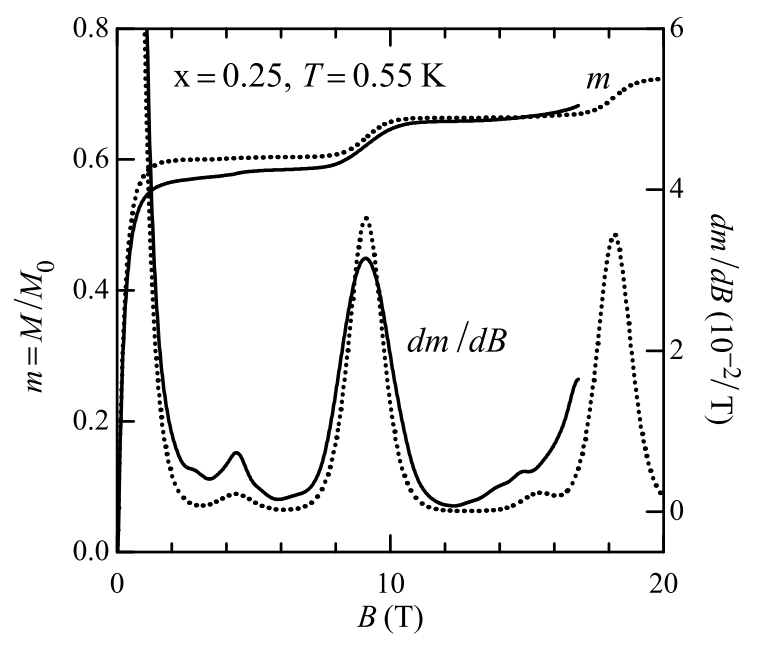

FIG. 8: Comparison between the dc data for $\mathrm{x}=0.25$ at $0.55 \mathrm{~K}$ (solid curves) with a simulation based on equilibrium theory for the single- $J$ model (dotted curves). The ordinate scales are for $m=M / M_{0}$, and $d m / d B$.

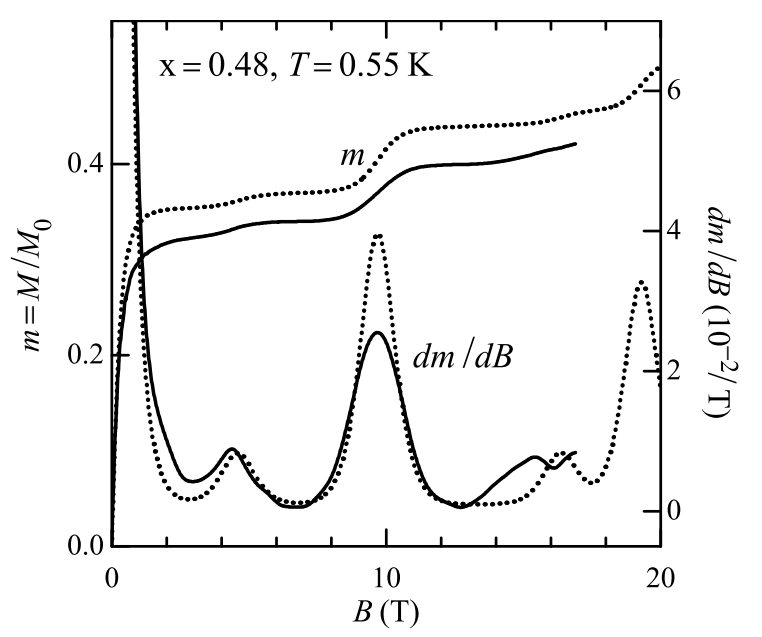

FIG. 9: Comparison between the dc data for $\mathrm{x}=0.48$ at $0.55 \mathrm{~K}$ (solid curves) with a simulation based on equilibrium theory for the single- $J$ model (dotted curves). The ordinate scales are for $m=M / M_{0}$, and $d m / d B$.

and simulation for $\mathrm{x}=0.48$ is only fair. The measured magnetization is somewhat smaller than expected, over the entire field range. In particular, the initial rise of the measured magnetization is smaller than in the simulation. This discrepancy suggests that the Mn ions have a slight tendency to bunch together, compared to a random distribution. ${ }^{-1}$ Also, the peak of $d m / d B$ near $15 \mathrm{~T}$ is at a lower field than predicted for the third MST from the quartets. The reason for this behavior is unclear. In our view, despite these discrepancies the agreement between experiment and theory is still reasonable. 


\section{DIFFERENTIAL SUSCEPTIBILITY IN PULSED FIELDS}

Pulsed-field data for $d M / d B$ were taken on powder samples with $\mathrm{x} \approx 0.50$ (from the same product as samples 5 and $5^{*}$ ), $\mathrm{x} \approx 0.22$ (same product as 2 and $2^{*}$ ), and $\mathrm{x} \approx 0.16$ (same product as $1^{*}$ ). As noted in Sec. [V] each sample was in direct contact with a liquid-helium bath, maintained at $T_{\text {bath }}=1.5 \mathrm{~K}$. However, such a direct contact does not ensure thermal equilibrium with the bath during the $7.4 \mathrm{~ms}$ pulse.

The pulsed-field data are presented next. Nonequilibrium features are pointed out, and many of them are then interpreted. However, some aspects of the nonequilibrium behavior are still not fully understood.

\section{A. Experimental Results in Pulsed Fields}

Figure 10 shows some $d M / d B$ data for $\mathrm{x} \approx 0.50$. Part (a) of this figure shows an "up trace" (increasing $B$ ) and a "down trace" (decreasing $B$ ) obtained during the same field pulse. Part (b) gives an expanded view of a portion of the down trace.

Results for $\mathrm{x} \approx 0.22$ are shown in Fig. 11(a). This figure covers the wide range of $d M / d B$ values that is required to display the large hysteresis in low fields. Data taken during the same field pulse, but which cover a much narrower range of $d M / d B$ values, are shown in Fig. 11] ( b).

Results obtained for $\mathrm{x} \approx 0.16$ during a pulse with a maximum magnetic field $B_{\max }=17 \mathrm{~T}$ are shown in Fig. 12(a). Figure 12(b) shows the decreasing-field portion of a trace obtained during another field pulse for which the maximum field was $34 \mathrm{~T}$. [Unlike all the other pulsed-field data in the present paper, the data in Fig. 12(b) have not been corrected for the monotonic background, because no "background shot" (with the sample out of the pickup coils) was taken in this case.] Figure 13 shows an expanded, and slightly smoother, view of the field-down portion of the Fig. 12(a).

\section{B. Discussion of non-equilibrium effects}

The pulsed-field data do not provide any new information about the exchange constant $J$. However, these data show interesting non-equilibrium effects. The discussion below focuses primarily on: 1) those features of the data that indicate the absence of thermal equilibrium; 2) the change of the non-equilibrium behavior with the Mn concentration $\mathrm{x}$; and 3) physical mechanisms that can give rise to such non-equilibrium effects.

$$
\text { 1. } \mathrm{x} \approx 0.50
$$

Consider first those pulsed-field results in Fig. 10 that are below $15 \mathrm{~T}$. There is a prominent peak near $10 \mathrm{~T}$. It stands out more clearly in the down trace (decreasing $B$ ) than in the up trace. This peak corresponds to the first MST from pairs. The down trace also shows a smaller peak just below $5 \mathrm{~T}$. This smaller peak corresponds to the first MST from the quartets. Although both of these peaks were also observed in the dc data for the equilibrium magnetization [Fig. 6(b)], at least two features of the pulsed-field data indicate departures from equilibrium with the helium bath, at $T_{\text {bath }}=1.5 \mathrm{~K}$. First, the up and down traces below $15 \mathrm{~T}$ are different, i.e., there is an hysteresis in this field range. Second, the widths of both the peak near $10 \mathrm{~T}$ and near $5 \mathrm{~T}$ are substantially smaller than the equilibrium width at the bath temperature.

The "width" of a MST will always refer to the full width at half maximum of the associated peak in $d M / d B$. The various contributions to the equilibrium width were discussed in Ref. 1. Often, temperature broadening is important. The thermal width at the temperature $T$ is

$$
(\delta B)_{T}=3.53 k_{\mathrm{B}} T / g \mu_{\mathrm{B}}
$$

Because non-thermal broadening mechanisms are also present in equilibrium, the thermal width is a lower limit for the actual equilibrium width. The calculated thermal width, $(\delta B)_{T}=3.9 \mathrm{~T}$ for the actual bath temperature $T_{\text {bath }}=1.5 \mathrm{~K}$, is shown in Fig. 10(b).

To obtain the experimental width it is necessary to choose a baseline for the peak in $d M / d B$. Such a choice is not always obvious. In Fig. 10(b), two possible choices for the peak near $10 \mathrm{~T}$ are indicated by the dashed lines 1 and 2. Baseline 1 leads to a full width at half maximum of 2.2 T. Baseline 2 leads to a width of $1.9 \mathrm{~T}$. On this basis we conclude that the experimental width is substantially smaller than the equilibrium width.

The choice of a baseline for the peak near $5 \mathrm{~T}$ is also not obvious. The particular choice shown as a dashed line leads to a width of $1.2 \mathrm{~T}$. Although other choices may lead to a larger experimental width, it seems that any reasonable choice will lead to a width that is smaller than the thermal width. Thus, the experimental widths of both of the peaks in Fig. 10(b) are smaller than the width that would have occurred had the sample been in equilibrium with the helium bath.

Figure 10 also shows the second MST from pairs, near $20 \mathrm{~T}$. This peak is broader than the first peak from the pairs. A significant feature of this peak is that it is asymmetric. As a function of time, the rise of $d M / d B$ as the peak is approached is faster than the fall after passing through the peak. This asymmetry is observed in both increasing and decreasing $B$. An asymmetry of this type is expected from models that assume an inadequate heat flow (Sec. IIIB). The data in Fig. 10] therefore suggest that the non-equilibrium behavior for $\mathrm{x} \approx 0.50$ is due to inadequate sample-to-bath heat flow.

The IHF scenario can also account for the hysteresis below about $15 \mathrm{~T}$. When the thermal contact with the bath is poor, strong magneto-caloric effects are expected from the singles (clusters with $n=1$ ). These effects are analogous to those involved in the cooling of a paramag- 
net by adiabatic demagnetization (and warming by adiabatic magnetization). Of course, the actual processes in the present case are not truly adiabatic, because there is some heat flow between the sample and the bath. In our view, no feature of the data in Fig. [10]requires CR for its explanation. The observed non-equilibrium effects seem to be explainable by an inadequate sample-to-bath heat flow.

Another issue (not directly related to the nonequilibrium behavior) involves the third MST from pairs, seen in Fig. 10 near 30 T. Compared to the first and second MST's from pairs, the third MST is less well defined. Specifically, $d M / d B$ hardly decreases on the high-field side of the third MST. This behavior is explained by the three small MST's from quartets, triplets, and quintets that are expected between the third and fourth MST's from pairs (see the MST's near $b=7$ in Fig. 33). For $\mathrm{x}=0.5$ the predicted combined size of these three small MST's is comparable to the size of one MST from pairs.

\section{Non-equilibrium behavior for $\mathrm{x} \approx 0.16$}

The absence of thermal equilibrium with the helium bath is very evident in the pulsed-field data for $\mathrm{x} \approx 0.16$.

(i) A pronounced hysteresis is seen in Fig. 12 (a).

(ii) In the down portion of the pulse (see Fig. 13) the widths of the peaks near 10,5 , and $2.7 \mathrm{~T}$ are $1.4,0.9$, and $0.6 \mathrm{~T}$, respectively. These values are small compared to a thermal width of $3.9 \mathrm{~T}$ at $T_{\text {bath }}=1.5 \mathrm{~K}$. A width that is smaller than the thermal width implies a non-equilibrium behavior.

(iii) The peak near $5 \mathrm{~T}$ is very pronounced in the pulsedfield data shown in Figs. 12 (a) and 13] but is barely seen in dc data on a sample from the same product. The dc data, shown in Fig. 14] should be representative of equilibrium behavior. Therefore, the pronounced peak in the pulsed field data is regarded as a non-equilibrium effect.

(iv) The second MST from pairs, near $18 \mathrm{~T}$, is barely observed in Fig. 12(b), and the third MST from pairs, near $27 \mathrm{~T}$, is totally absent. The corresponding field-up trace (not shown) exhibits a similar behavior. Once again the pulsed field data are contrasted with the equilibrium magnetization data in Fig. 14. The latter data, which extend up to $17.5 \mathrm{~T}$, show a significant portion of the second MST from pairs, and they indicate that in equilibrium the sizes of the second and first MST's from pairs are comparable. That is, unlike the behavior in pulsed fields, the second MST is not small compared to the first. In equilibrium, all MST's from pairs are expected to be comparable, which is inconsistent with the results of Fig. 12(b).
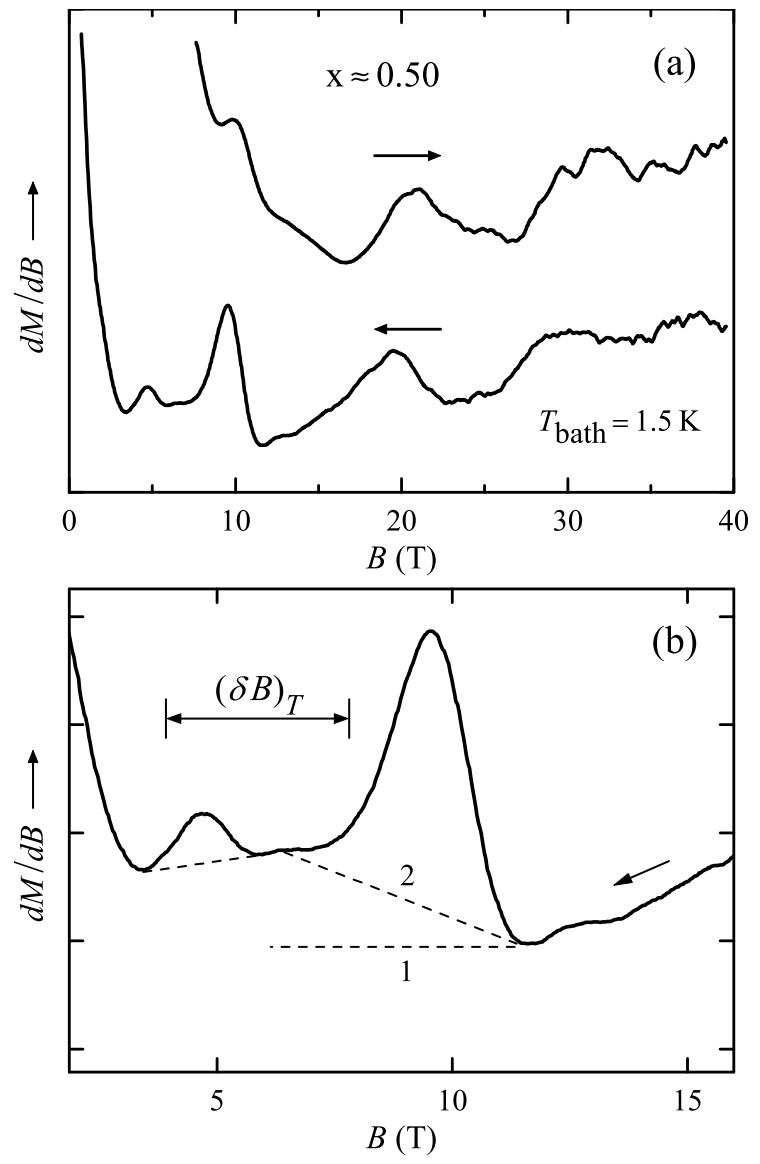

FIG. 10: (a) Pulsed-field data of $d M / d B$ for $\mathrm{x} \approx 0.50$ in both increasing $B$ ("up") and decreasing $B$ ("down"). The up and down traces are shifted vertically relative to each other. (b) Expanded view of a portion of the down trace. The calculated thermal width $(\delta B)_{T}$ at the bath temperature $T_{\text {bath }}=1.5 \mathrm{~K}$ is indicated. Dashed lines show some choices of baselines used to obtain the experimental widths of two peaks.

\section{IHF and CR Scenarios for $\mathrm{x} \approx 0.16$}

The large hysteresis at low fields [Fig. [12(a)] is not uncommon in pulsed field experiments on diluted magnetic materials ${ }^{28.37}$ In the present case the hysteresis is largely due to the slow response of the singles to the rapidly changing $B$. In the up portion of the pulse the alignment of the spins of the singles is not completed until $B$ is above $10 \mathrm{~T}$. In the down portion of the pulse these spins remain largely aligned until $B$ drops below $1 \mathrm{~T}$. For $\mathrm{x} \approx 0.16$, the majority of the spins $(70 \%$ for a random distribution) are singles, so that the hysteresis is very pronounced.

MST's from pairs and larger clusters stand out more clearly in the down portion of the field pulse because the singles remain largely aligned until $B$ drops below $1 \mathrm{~T}$. For this reason the field-down portion of the traces in Figs. 13 and 12(b) is discussed first. 

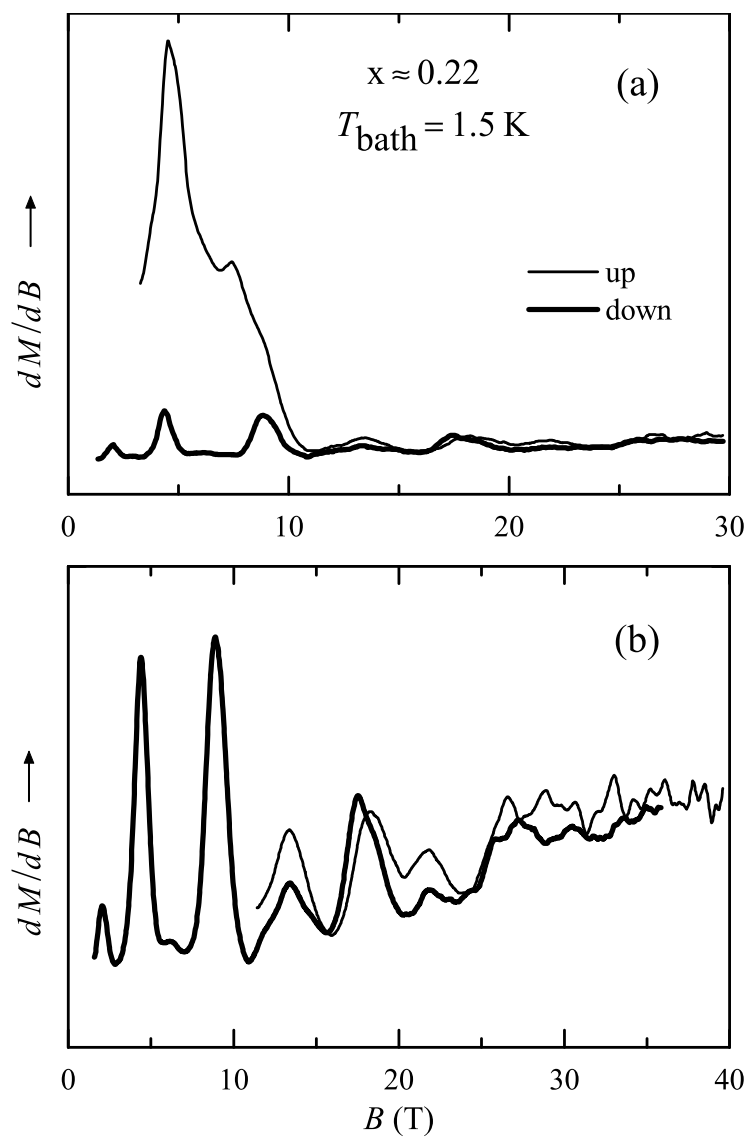

FIG. 11: Pulsed-field data of $d M / d B$ for $\mathrm{x} \approx 0.22$. (a) Overall view, showing the large hysteresis below about $10 \mathrm{~T}$. (b) Expanded view of the field-up and field-down traces obtained during the same field-pulse. The low-field portion of the up trace is excluded. The up and down traces are not shifted vertically relative to each other.

Spin-lattice relaxation times are often longer for lower $\mathrm{x}$, which is the likely cause of the more pronounced nonequilibrium behavior for $\mathrm{x} \approx 0.16$ compared to $\mathrm{x} \approx 0.50$. An important issue in the data interpretation is whether the spin-lattice relaxation is fast enough to maintain equilibrium within the sample. In that case the IHF scenario would apply (see Sec. 世B 2). An alternative is a more severe non-equilibrium behavior which is better described by the CR scenario, including single spin flips near level crossings of excited states (Sec. IIIB 3) 37.38

Below we consider both the IHF and CR scenarios for the down portion of the pulse. The preferred scenario cannot be chosen on the basis of the fields at which the MST's occur, because the differences are often small compared to the experimental accuracy. However, the two scenarios lead to different relative sizes of the peaks in $d M / d B$. The poor agreement of the observed relative sizes with those predicted by the IHF scenario will suggest that the CR scenario is preferable for this $\mathrm{Mn}$ concentration.
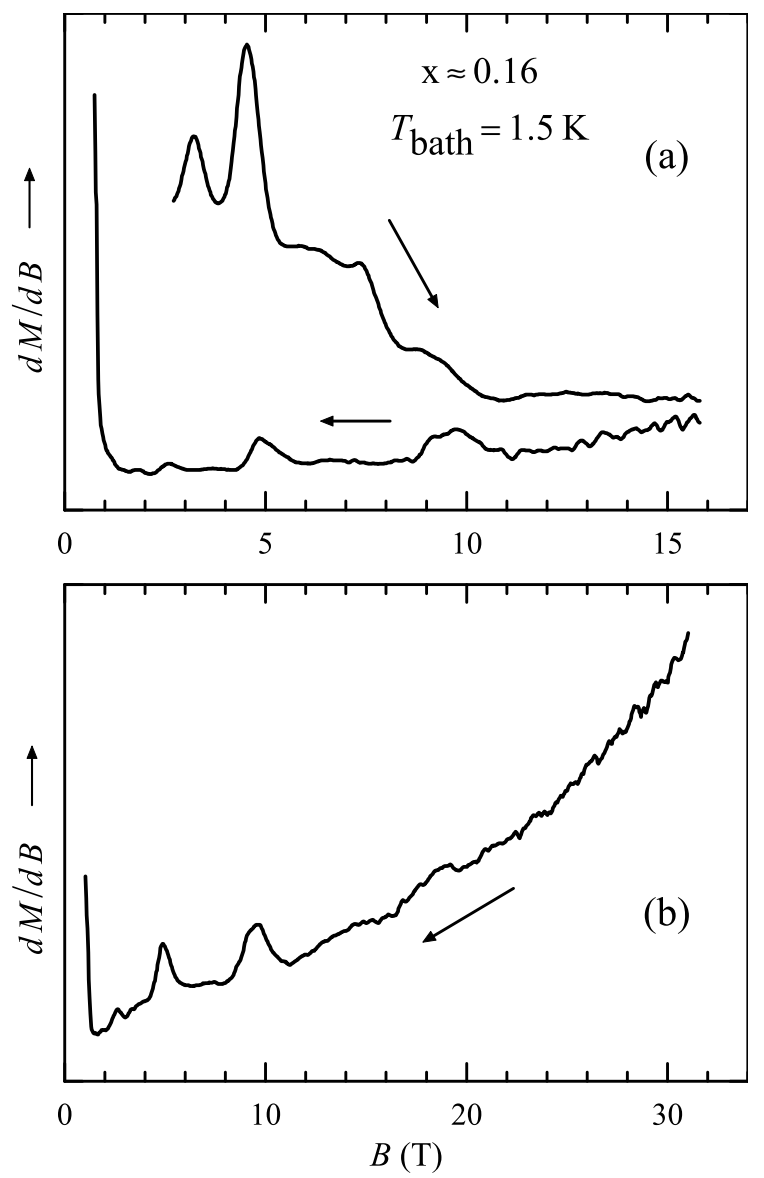

FIG. 12: (a) Results for $\mathrm{x} \approx 0.16$, obtained during a field pulse with a maximum field $B_{\max }=17 \mathrm{~T}$. The up and down traces are not shifted vertically relative to each other. (b) The down portion of a trace for $\mathrm{x} \approx 0.16$, obtained in another pulse with $B_{\max }=34 \mathrm{~T}$. This particular trace, unlike all others, is not corrected for background.

Consider first the IHF scenario. In this scenario the magnetization of each cluster type is the equilibrium magnetization at $T_{s}$. For the low $T_{s}$ indicated by the small widths of the observed MST's (in the down portion of the pulse), this magnetization is that of the ground state. The largest peak in Fig. 12 just below $10 \mathrm{~T}$, is mainly due to the first MST from pairs. The second largest peak, near $5 \mathrm{~T}$ is the first MST from quartets. It is predicted to occur at a field which is 0.48 times that of the first peak for pairs. The second $d M / d B$ peak from quartets should be at a field which is $2 \%$ higher than the first peak from pairs. The structure of the peak near $10 \mathrm{~T}$ is possibly due to a superposition of these two MST's, although it is much wider than $2 \%$.

In the IHF scenario the field at the first MST for finite chains with even $n$ can be estimated from the energy levels given in Ref. 20. For $n$ between 4 and 10, this field is nearly proportional to $1 / n$. A similar approximate dependence on $n$ holds for AF rings (closed chains) $\stackrel{2}{2}$ On 


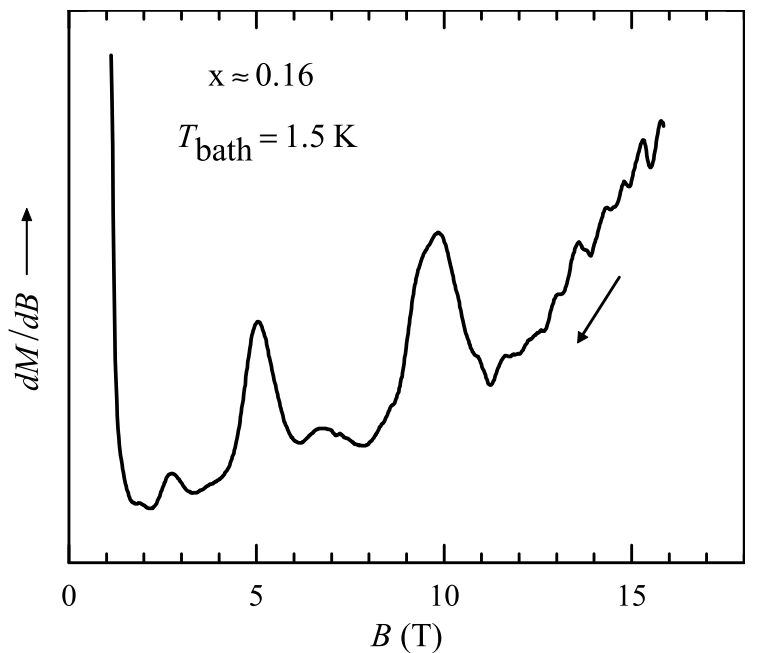

FIG. 13: An expanded, and slightly smoother, view of the field-down portion of Fig. [12 a).

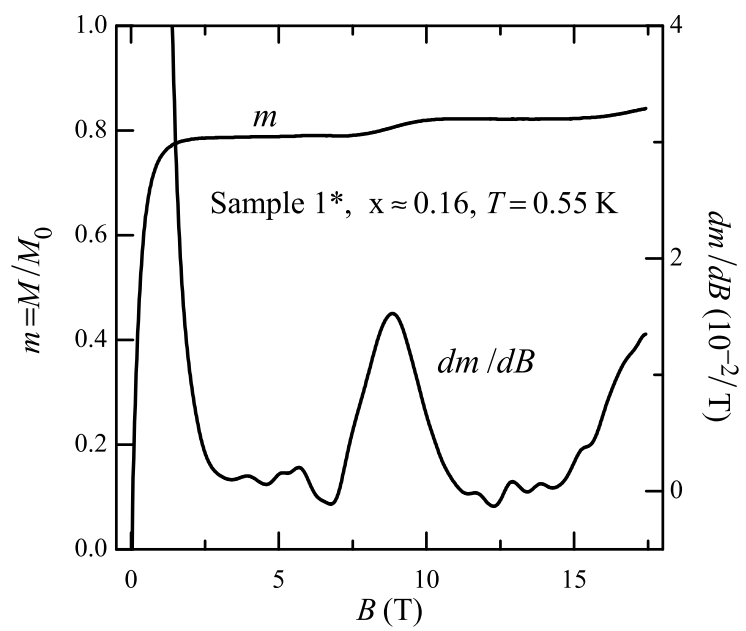

FIG. 14: The dc magnetization $M$ at $0.55 \mathrm{~K}$ for $\mathrm{x} \approx 0.16$ (sample $1^{*}$ ). Also shown is the numerical derivative $d m / d B$. In the text, some features of these equilibrium-magnetization data are contrasted with pulsed-field data on a similar sample (Figs. 12 and 13).

this basis the small sharp peak near $2.7 \mathrm{~T}$ (in Fig. 12) is due to octets or sextets. There is also an indication of a small peak near $2.0 \mathrm{~T}$, which would be attributed to larger clusters.

Although the IHF scenario accounts for the fields of many of the observed MST's, this scenario is very questionable for this Mn concentration. Assuming that the Mn cations are randomly distributed, the number of quartets for $\mathrm{x}=0.16$ is smaller than the number of pairs by a factor of 39 . For sextets and octets the factors are $1.5 \times 10^{3}$ and $6 \times 10^{4}$, respectively. Therefore, unless the deviations from random distribution are extremely large, it should not have been possible to observe MST'S from sextets or octets if the behavior followed the IHF scenario. The first MST from quartets might have been detectable, but it should have been very small compared to the MST from pairs. This was not the case in the pulse field experiments (Figs. 12 and 13). As predicted, the equilibrium data in Fig. 14, for nearly the same $\mathrm{x}$, indicate that the first MST from quartets is much less pronounced than the first MST from pairs.

In Fig. 12(b) the first MST from pairs stands out clearly but the second MST from pairs is barely visible, and the third is totally absent. These results also are not well understood within the IHF scenario, although some indication of such a behavior appeared in simulations by Nakano and Miyashita for iron clusters with ring structure 31

In the CR scenario the MST's in Figs. 12(b) and 13 (both for decreasing B) are interpreted as follows. The peak just below $10 \mathrm{~T}$ is the first fundamental peak $\mathrm{P}_{1}$, with some contributions from $\mathrm{P}_{2 / 2}, \mathrm{P}_{3 / 3}$, etc. The peak at $5 \mathrm{~T}$ is the second harmonic peak $\mathrm{P}_{1 / 2}$. The small peak at $2.7 \mathrm{~T}$ is the third-harmonic peak $\mathrm{P}_{1 / 3}$ or the fourth-harmonic peak $\mathrm{P}_{1 / 4}$. The broad peak near $6.6 \mathrm{~T}$ is possibly $\mathrm{P}_{2 / 3}$. Because this interpretation uses only pairs and singles, ${ }^{37}$ it is not open to objections based on the low populations of quartets and larger clusters. The CR scenario also accounts for some features of the up trace in Fig. 12(a). The large peak near $5 \mathrm{~T}$ is attributed to the CR process of the type shown in Fig. 4(b), except that the directions of both spin flips are reversed. This process allows the singles to relax toward a state with a higher magnetization. The magnetization of the pairs also increases by this process.

The process in Fig. 4(b) involves only one single and one pair, and is the simplest CR process between singles and pairs. It accounts for the $\mathrm{P}_{1 / 2}$. More complicated CR processes can lead to other "harmonic peaks," such as $\mathrm{P}_{1 / 3}, \mathrm{P}_{2 / 3}, \mathrm{P}_{3 / 4}$. These peaks are expected to be smaller than $\mathrm{P}_{1 / 2}$. The peak $\mathrm{P}_{1 / 3}$ may involve processes such as a CR between a pair and two singles, or between a pair and a single which undergoes a double spin flip. The peak $\mathrm{P}_{2 / 3}$ may involve a spin flip in a single and spin flips in two pairs, etc. In the up trace the observed peak at $3.2 \mathrm{~T}$, and the small peaks at 6.4 , and $7.4 \mathrm{~T}$ may correspond to the $\mathrm{P}_{1 / 3}, \mathrm{P}_{2 / 3}$ and $\mathrm{P}_{3 / 4}$ harmonics. The peak observed near $10 \mathrm{~T}$ is a superposition of the first fundamental peak from pairs, $\mathrm{P}_{1}$, and the harmonics $\mathrm{P}_{2 / 2}, \mathrm{P}_{3 / 3}$, etc.

As already noted, in Fig. 12(b) the second MST from pairs is barely visible, and the third is totally absent. These results suggest that for $\mathrm{x} \approx 0.16$, the spin relaxation for pairs in fields above $15 \mathrm{~T}$ is very slow compared to a millisecond. As $B$ sweeps through a region where a MST from pairs should have occurred, the pairs are unable to relax towards the new ground state. We speculate that the slow spin relaxation for pairs is mainly due to a reduction of $\mathrm{CR}$ between pairs and singles, and that this reduction is related to the saturation of the singles in fields above $15 \mathrm{~T}$. CR between different pairs, or be- 
tween pairs and larger clusters, is expected to become slower as $\mathrm{x}$ decreases 41 Among the three samples, such $\mathrm{CR}$ processes should be least efficient for $\mathrm{x} \approx 0.16$.

\section{Non-equilibrium behavior for $\mathrm{x} \approx 0.22$}

Non-equilibrium behavior is also evident for $\mathrm{x} \approx 0.22$. The large low-field hysteresis for this Mn concentration, in Fig. [11(a), is somewhat similar to the hysteresis in Fig. 12(a) for $\mathrm{x} \approx 0.16$. In the up trace, the large peak near $4.5 \mathrm{~T}$ is identified as the $\mathrm{P}_{1 / 2}$ peak, and is attributed to the cross relaxation process in Fig. 4(b), with the arrows reversed.

The down trace in Fig. 11(b) exhibits large peaks near $8.9 \mathrm{~T}$ and $4.4 \mathrm{~T}$ approximately, and a small peak near $2.1 \mathrm{~T}$. The widths at half height of these peaks, 1.3, 0.9, and $0.7 \mathrm{~T}$, respectively, are all much smaller than thermal width of $3.9 \mathrm{~T}$ at $T_{\text {bath }}$. These widths, which are similar to those for $\mathrm{x} \approx 0.16$, indicate non-equilibrium behavior.

The largest peak in Fig.11(b), at 8.9 T, is undoubtedly the first fundamental peak from pairs, $\mathrm{P}_{1}$. The second fundamental peak $\mathrm{P}_{2}$ is also observed near $18 \mathrm{~T}$. The $\mathrm{CR}$ scenario predicts large second-harmonic peaks $\mathrm{P}_{1 / 2}$, $\mathrm{P}_{3 / 2}, \mathrm{P}_{5 / 2}$ at $4.5,13.4$, and $22.3 \mathrm{~T}$, respectively. These fields are close to $4.4,13.4$, and $21.8 \mathrm{~T}$, of large peaks in Fig. 11(b). The peak observed near $2.1 \mathrm{~T}$ is consistent with $\mathrm{P}_{1 / 4}$. It is possible, but far from certain, that the small peaks at 6.2 and $12.0 \mathrm{~T}$ are $\mathrm{P}_{2 / 3}$ and $\mathrm{P}_{4 / 3}$.

The main difference between $\mathrm{x} \approx 0.22$ and $\mathrm{x} \approx 0.16$ is that for the higher $\mathrm{Mn}$ concentration there are still prominent MST's above $10 \mathrm{~T}$. For $\mathrm{x} \approx 0.50$ the second and third MST's from pairs are even more pronounced [see Fig. 10(a)]. These results suggest that at these fields the spin relaxation rate for pairs increases rapidly with Mn concentration. We tentatively attribute this trend to the expected increase with $\mathrm{x}$ of the efficacy of $\mathrm{CR}$ processes involving pairs, and pairs and larger clusters.

\section{Summary of the analysis of non-equilibrium behavior}

A definitive interpretation of the observed nonequilibrium behavior in pulsed fields is still lacking. However, it appears that for $\mathrm{x} \approx 0.50$ the non-equilibrium behavior is better explained by the IHF scenario. For $\mathrm{x} \approx 0.16$ and $\mathrm{x} \approx 0.22$ the data are better explained by spin flips associated with $\mathrm{CR}$ and with level crossings. The data suggest that at the high magnetic fields where the magnetization of singles is saturated, the spin relaxation rate for pairs increases rapidly with increasing $\mathrm{x}$. This increase is tentatively attributed to the $\mathrm{x}$ dependence of CR processes involving pairs.

\section{Acknowledgments}

We thank R. Muccillo (USP) and J. A. Adario (MIT) for X-rays measurements. The work in Brazil was supported by FAPESP (Fundação de Amparo à Pesquisa do Estado de São Paulo, Brazil) under contract number 99/10359-7. The authors APF, NFOJ and VB acknowledge support from CNPq (Conselho Nacional de Desenvolvimento Científico e Tecnológico, Brazil). Travel funds for YS were provided by FAPESP.
* Electronic address: apaduan@if.usp.br

† Electronic address: vbindilatti@if.usp.br

¥ Electronic address: yshapira@granite.tufts.edu

1 Y. Shapira and V. Bindilatti, J. Appl. Phys. 92, 4155 (2002). URL http://link . aip.org/link/?jap/92/4155

2 D. Gatteschi, J. Phys. Chem. B 104, 9780 (2000); URL http://dx.doi.org/10.1021/jp001644w D. Gatteschi and R. Sessoli, in Magnetism: Molecules to Materials III, J. S. Miller and M. Drillon, eds. (Wiley-VCH, Weinheim, 2002), pp. 63-108.

3 S. A. Crooker, D. G. Rickel, S. K. Lyo, N. Samarth, and D. D. Awschalom, Phys. Rev. B 60, R2173 (1999). URL http://link.aps.org/abstract/PRB/v60/p2173

4 L. J. de Jongh and A. R. Miedema, Adv. Phys. 23, 1 (1974).

5 M. Steiner, J. Villain, and C. G. Windsor, Adv. Phys. 25, 87 (1976).

6 R. L. Carlin, Magnetochemistry (Springer, Berlin, 1986).

7 O. Kahn, Molecular Magnetism (Wiley, New York, 1993).

8 M. T. Hutchings, G. Shirane, R. J. Birgeneau, and S. L. Holt, Phys. Rev. B 5, 1999 (1972). URL http://link.aps.org/abstract/PRB/v5/p1999

9 L. R. Walker, R. E. Dietz, K. Andres, and S. Darack, Solid State Commun. 11, 593 (1972). URL http://dx.doi.org/10.1016/0038-1098(72)90468-1

10 P. S. Peercy, B. Morosin, and G. A. Samara, Phys. Rev. B 8, 3378 (1973). URL http://link.aps.org/abstract/PRB/v8/p3378

11 M. N. Braud, M. Couzi, N. B. Chanh, C. Courseille, B. Gallois, C. Hauw, and A. Meresse, J. Phys. Condens. Matter 2, 8209 (1990). URL http://stacks.iop.org/0953-8984/2/8209

12 I. Peral, G. Madariaga, A. Perez-Etxebarria, and T. Breczewski, Acta Cryst. B 56, 215 (2000). URL http://dx.doi.org/10.1107/S0108768199013622

13 C. Dupas and J. P. Renard, Phys. Rev. B 18, 401 (1978). URL http://link . aps .org/abstract/PRB/v18/p401

14 I. Harada, C. Suzuki, and T. Tonegawa, J. Phys. Soc. Jpn. 49, 942 (1980).

15 J. C. Schouten, K. Kopinga, and W. J. M. de Jonge, J. Phys. C: Solid State Phys. 15, 783 (1982). URL http://stacks .iop.org/0022-3719/15/783

16 J. C. Bonner and M. E. Fisher, Phys. Rev. 135, A640 (1964). URL http://link.aps.org/abstract/PR/v135/pA640

17 J. B. Parkinson and J. C. Bonner, Phys. Rev. B 32, 4703 (1985). URL http://link.aps.org/abstract/PRB/v32/p4703 
18 For early works see: R. J. Elliott and B. R. Heap, Proc. R. Soc. London Ser. A 265, 264 (1962); and M. E. Fisher and J. W. Essam, J. Math. Phys. 2, 609 (1961). The relevant equation is reproduced in Ref. 1 as Eq. (18). An equivalent expression is given in Ref. 14, among others.

19 X. Gratens, V. Bindilatti, E. terHaar, N. F. Oliveira, Jr., Y. Shapira, and F. C. Montenegro, Phys. Rev. B 64, 214424 (2001). URL http://link.aps.org/abstract/PRB/v64/e214424

20 See, e.g., J. Lou, S. Qin, T.-K. Ng, and Z. Su, Phys. Rev. B 65, 104401 (2002). URL http://link.aps.org/abstract/PRB/v65/e104401

21 Taking the sum up to $n=\infty$ may seem inconsistent with the assumed short chain model. However, for the low $\mathrm{x}$ considered here (below about 0.5), the contribution to the sum in Eq. (8) from chains that are not short is negligible.

22 J. R. Friedman, M. P. Sarachik, J. Tejada, and R. Ziolo, Phys Rev. Lett. 76, 3830 (1996). URL http://publish.aps.org/abstract/PRL/v76/p3830

23 L. Thomas, F. Lionti, R. Ballou, D. Gatteschi, R. Sessoli, and B. Barbara, Nature 383, 145 (1996).

24 E. M. Chudnovsky and J. Tejada, Macroscopic Quantum Tunneling of the Magnetic Moment (Cambridge, New York, 1998).

25 B. Barbara and L. Gunther, Physics World $12 \quad(3), \quad 35 \quad$ (1999). URL http://physicsweb.org/article/world/12/3/12

26 Y. Shapira, S. Foner, D. Heiman, P. A. Wolff, and C. R. McIntyre, Solid State Commun. 71, 355 (1989). URL http://dx.doi.org/10.1016/0038-1098(89)90770-9

27 V. Bindilatti, T. Q. Vu, and Y. Shapira, Solid State Commun. 77, 423 (1991). URL http://dx.doi.org/10.1016/0038-1098(91) 90229-0

28 S. Foner, Physica B 201, 13 (1994). URL http://dx.doi.org/10.1016/0921-4526(94)91043-X

29 Y. Shapira, M. T. Liu, S. Foner, C. E. Dube, and P. J. Bonitatebus, Phys. Rev. B 59, 1046 (1999). URL http://link.aps.org/abstract/PRB/v59/p1046

30 I. Chiorescu, W. Wernsdorfer, A. Muller, H. Bogge, and B. Barbara, Phys. Rev. Lett. 84, 3454 (2000); URL http://link.aps.org/abstract/PRL/v84/p3454 I. Chiorescu, W. Wernsdorfer, A. Muller, S. Miyashita, and B. Barbara, Phys. Rev. B 67, 020402 (2003). URL http://link.aps.org/doi/10.1103/PhysRevB.67.020402

31 H. Nakano and S. Miyashita, J. Phys. Soc. Jpn.
70, 2151 (2001); K. Saito and S. Miyashita, ibid. 70, 3385 (2001); H. Nakano and S. Miyashita, J. Phys. Chem. Solids 63, 1519 (2002). URL http://dx.doi.org/10.1016/S0022-3697(02)00068-9

32 Y. Shapira, M. T. Liu, S. Foner, R. J. Howard, and W. H. Armstrong, Phys. Rev. B 63, 094422 (2001). URL http://link.aps.org/abstract/PRB/v63/e094422

33 O. Waldmann, R. Koch, S. Schromm, P. Muller, I. Bernt, and R. W. Saalfrank, Phys. Rev. Lett. 89, 246401 (2002); URL http://link.aps.org/abstract/PRL/v89/e246401 90, 229904(E) (2003). URL http://link.aps.org/abstract/PRL/v90/e229904

34 Y. Inagaki, T. Asano, Y. Ajiro, Y. Narumi, K. Kindo, A. Cornia, and D. Gatteschi, J. Phys. Soc. Jpn. 72, 1178 (2003).

35 W. P. Wolf, Phys. Rev. 115, 1196 (1959). URL http://link.aps.org/abstract/PR/v115/p1196

36 See, for example: K. Amaya, Y. Tokunaga, R. Yamada, Y. Ajiro, and T. Haseda, Phys. Lett. A 28, 732 (1969); URL http://dx.doi .org/10.1016/0375-9601(69)90589-1 F. Varret, Y. Allain, and A. Miedan-Gros, Solid State Commun. 14, 17 (1974); URL http://dx.doi.org/10.1016/0038-1098(74)90222-1 and D. L. Meier, M. Karnezos, and S. A. Friedberg, Phys. Rev. B 28, 2668 (1983). URL http://link.aps.org/abstract/PRB/v28/p2668

37 Y. Ajiro, T. Asano, H. Aruga-Katori, T. Goto, K. Koui, K. Kamishima, and H. Ikeda, Physica B 246-247, 222 (1998). URL http://dx.doi.org/10.1016/S0921-4526(98)00013-1

38 W. Wernsdorfer, S. Bhaduri, R. Tiron, D. N. Hendrickson, and G. Christou, Phys. Rev. Lett. 89, 197201 (2002). URL http://link.aps.org/abstract/PRL/v89/e197201

39 R. Giraud, W. Wernsdorfer, A. M. Tkachuk, D. Mailly, and B. Barbara, Phys. Rev. Lett. 87, 057203 (2001). URL http://link.aps.org/abstract/PRL/v87/e057203

40 J. H. van Vleck, Phys. Rev. 59, 724 (1941). URL http://link.aps.org/abstract/PR/v59/p724

41 A. Abragam and B. Bleaney, Electron Paramagnetic Resonance of Transition Ions (Oxford, London, 1970).

42 X. Y. Dong, S. Ahmed, and A. R. McGurn, Phys. Rev. B 37, 7575 (1988). URL http://link.aps.org/abstract/PRB/v37/p7575 